\title{
Joint Channel Estimation and Equalization for Index-Modulated Spectrally Efficient Frequency Division Multiplexing Systems
}

\author{
Yunsi Ma, Student Member, IEEE, Nan Wu, Member, IEEE, Weijie Yuan, Member, IEEE, \\ Derrick Wing Kwan Ng, Senior Member, IEEE, and Lajos Hanzo, Fellow, IEEE
}

\begin{abstract}
Spectrally efficient frequency division multiplexing (SEFDM) relying on index modulation (IM) has emerged as a promising multicarrier technique. In this paper, we develop a joint channel estimation and equalization method based on factor graphs for SEFDM-IM signaling over frequency-selective fading channels. By approximating the interference in the frequency domain, we reformulate the problem to obey a linear state-space model and construct a multi-layer factor graph. To support a reconfigurable architecture, non-orthogonal demodulation is adopted and the colored noise encountered is approximated by a complex auto-regressive (CAR) model. For deriving a low-complexity parametric Gaussian message passing (GMP)based method, we exploit an expectation propagation (EP)-based technique for approximating the discrete $a$ posteriori distributions of the transmitted symbols in a Gaussian form. To further simplify the result, variational message passing (VMP) is applied to an equivalent soft node to obtain a Gaussian form. Moreover, we also derive the Cramér-Rao lower bound (CRLB) in closedform. The overall complexity only grows linearly with the number of subcarriers and logarithmically with the length of the channel's memory. Compared to its Nyquist signaling based counterpart, SEFDM-IM signaling relying on the proposed algorithm exhibits up to $25 \%$ higher bandwidth efficiency without any bit error rate (BER) performance degradation.
\end{abstract}

Index Terms-Spectrally efficient frequency division multiplexing, index modulation, channel estimation, complex-valued colored noise, variational message passing.

\section{INTRODUCTION}

Non-orthogonal transmission schemes having high spectral efficiency have been conceived for next generation wireless communication systems [1]-[9]. The spectrally efficient frequency division multiplexing (SEFDM) scheme of [10], [11] also belongs to the family of non-orthogonal multicarrier techniques, which has been investigated in optical communications

This work was supported by the "National Science Foundation of China (NSFC)" (Grant No.61571041,61971041). D. W. K. Ng is supported by funding from the UNSW Digital Grid Futures Institute, UNSW, Sydney, under a cross-disciplinary fund scheme and by the Australian Research Council's Discovery Project (DP190101363). L. Hanzo would like to acknowledge the financial support of the Engineering and Physical Sciences Research Council projects EP/N004558/1, EP/P034284/1, EP/P034284/1, EP/P003990/1 (COALESCE), of the Royal Society's Global Challenges Research Fund Grant as well as of the European Research Council's Advanced Fellow Grant QuantCom. (Corresponding Author: Nan Wu.)

Y. Ma and N. Wu are with the School of Information and Electronics, Beijing Institute of Technology, Beijing 100081, China (e-mail: yunsi@bit.edu.cn; wunan@bit.edu.cn).

W. Yuan and D. W. K. Ng are with the School of Electrical Engineering and Telecommunications, University of New South Wales, Sydney, NSW 2052, Australia (e-mail: weijie.yuan@unsw.edu.au; w.k.ng@unsw.edu.au).

L. Hanzo is with the School of Electronics and Computer Science, University of Southampton, Southampton SO17 1BJ, U.K. (e-mail: lh@ecs.soton.ac.uk).
[12], visible-light communication (VLC) [13], and in satellite systems [14]. By allowing the overlapping of the originally orthogonal subcarriers, SEFDM signaling becomes capable of significantly improving the spectral efficiency, at the cost of deliberately introducing intercarrier interference (ICI).

Unfortunately, its optimal maximum likelihood sequence (MLS) detection suffers from an excessive computational complexity [15]. To reduce the complexity, whilst still performing close to the optimal detector, numerous contributions have been focused on receiver design for SEFDM signaling [16]-[22]. The fixed-complexity sphere decoding (FSD) based equalizer combined with truncated singular value decomposition (TSVD) [16] and the iterative detector (ID) of [17] have been shown to be applicable to systems having a low number of subcarriers. By contrast, the fast Fourier transform (FFT)-based successive interference canceller (SIC) of [18] was conceived for SEFDM systems having a large number of subcarriers. However, soft information exchanging between the time- and frequency-domains still results in prohibitively high computational complexity. For effectively mitigating the ICI imposed by the non-orthogonality of SEFDM signaling, a separate frequency-domain zero-forcing (ZF)-based equalizer and a maximum a posteriori (MAP) sequential decoders were developed in [19] for zero-padding (ZP)-aided SEFDM systems. The above SEFDM receivers have assumed the availability of perfectly known channel state information (CSI). Due to the effect of strong inherent interference and owing to the ill-conditioning nature of SEFDM systems, only a few contributions have proposed channel estimators for SEFDM signaling over dispersive fading channels. In [20], a timedomain full channel estimator (FCE) based on zero-forcing (ZF) was proposed for SEFDM systems, which suffers from an ill-conditioning problem imposed by compressing the spacing of subcarriers. To eliminate this problem, a time-domain partial channel estimator (PCE) was developed in [21], which transmits pilots only on mutually orthogonal subcarriers. By contrast, in [22] three pilot-based frequency-domain channel estimation methods were designed for SEFDM systems at the cost of an additional interpolation operation or at the expense of introducing extra pilot symbol periods. However, the aforementioned channel estimators cannot obtain an acceptable performance.

To further improve spectral efficiency and energy efficiency, non-orthogonal transmission techniques have also been combined with index modulation (IM), e.g., [23]-[30]. Owing to 
the inevitable interference imposed by non-orthogonal signaling combined with IM, the complexity of the optimal maximum likelihood (ML) receiver increases exponentially both with the number of IM subblocks and with that of the transmitted bits per subblock. In [28], a block-based ML (BML) detector was proposed for mitigating the interferences, which relied on constructing a block diagonal equivalent channel matrix. Nevertheless, its complexity still grows exponentially with the length of the channel information response (CIR). To reduce the complexity of the receiver, the solutions in [29], [30] separated the interference mitigation relying on equalization from the IM detection. A minimum mean square error (MMSE)-based frequency-domain equalizer was employed in [29] for mitigating the interferences imposed by non-orthogonal signaling and then an ML detector was used for detecting the information bits. For SEFDM-IM signaling, the classical additional Gram-Schmidt orthonormalization was invoked for mitigating the effects of colored noise. Then, an MMSE-based equalizer and a log-likelihood ratio (LLR)-based IM detector having an expanded search space were invoked for eliminating the ICI and for detecting the transmitted bits, respectively. However, the aforementioned receivers have not exploited the potential performance gain of joint interference mitigation and IM detection. Moreover, the receivers conceived for non-orthogonal IM have typically assumed perfectly known CSI, which are not applicable to systems with unknown CSI.

Against this background, we propose a new joint channel estimation and equalization algorithm for SEFDM-IM systems communicating over frequency-selective fading channels. The main contributions of this paper are summarized as follows:

- To maintain compatible with typical multicarrier systems, we design a reconfigurable non-orthogonal demodulation architecture and then employ a complex autoregressive (CAR) model to deal with the resultant colored noise. Taking into account the dependencies between the SEFDM-IM subcarriers, we introduce an extended constellation including the deactivated symbols of zeros to explore the potential performance gain of joint ICI mitigation and IM detection. By ignoring the rather insignificant interference contributions, a Forney-style factor graph (FFG) having two subgraphs with scalar input is constructed based on the linear state-space model of SEFDM-IM equalization. Accordingly, a series of parametric message updating expressions constructed our FFGs are derived according to Gaussian message passing (GMP) rules, where the discrete distribution of the transmitted symbols can be approximated by a Gaussian form using expectation propagation (EP) rules by minimizing the Kullback-Leibler divergence (KLD) [31].

- We propose a low-complexity frequency-domain joint channel estimation and equalization algorithm for SEFDM-IM systems communicating over frequencyselective fading channels. To alleviate the potential error propagations imposed by channel estimation due to the deactivated subcarriers of zeros and improve the accuracy of channel estimates, we construct a multi-layer factor graph connected via channel variables on the subcarriers having the same indices between the different SEFDMIM symbols. Since applying belief propagation (BP) to the inner product node of channel estimation and equalization leads to excessive computational complexity, we resort to variational message passing (VMP) rules defined on an equivalent soft node to simplify the message updating expressions between the channel estimator and the equalizer into a Gaussian form. Combined with other diagonalized approximations and fast Fourier transform (FFT) operations, a parametric algorithm can be derived on FFG. Moreover, we also derive the Cramér-Rao lower bound (CRLB) for the proposed channel estimator in closed-form.

The rest of this paper is organized as follows. The system model of SEFDM-IM signaling over frequency-selective fading channels is given in Section II. In Section III, GMP-EP equalization is developed for SEFDM-IM systems under the assumption of having perfectly known CSI. In Section IV, the proposed GMP-EP-VMP joint channel estimation and equalization is derived. The performance of the proposed algorithms is evaluated by Monte Carlo simulations in Section V. Finally, our conclusions are drawn in Section VI.

Notations: Boldface capital and lowercase letters denote matrices and vectors, respectively. The operations $(\cdot)^{*},(\cdot)^{T}$, $(\cdot)^{H},(\cdot)^{-1}$, and $\operatorname{tr}(\cdot)$ denote the complex conjugate, transpose, conjugate transpose, matrix inverse, and trace operator, respectively. The operator $\odot$ denotes element-wise product. $\mathbf{I}_{L}$ and $\mathbf{0}_{L}$ are the identity matrix and all-zeros matrix with size $L \times L$, respectively. $\mathcal{D}(\mathbf{x})$ represents a diagonal matrix constructed from the vector $\mathbf{x} . \mathcal{C N}\left(m_{x}, V_{x}\right)$ denotes a complex Gaussian distribution of variable $x$ with mean $m_{x}$ and variance $V_{x}$, the probability density function of which is represented as $g_{\mathcal{C}}\left(m_{x}, V_{x} ; x\right)$. The operator $\propto$ denotes equality up to a constant normalization factor. $\mathbb{E}\{\cdot\}$ denotes expectation operation. $\mathbb{N}$ is the field of natural number. $\operatorname{sinc}(\cdot)$ represents the sinc function, i.e., $\operatorname{sinc}(x)=\sin (\pi x) /(\pi x)$, and $\tanh (\cdot)$ is the tangent function. $\left(\begin{array}{l}N \\ K\end{array}\right)$ denotes the binomial coefficient and $\lfloor\cdot\rfloor$ is the floor function.

\section{SySTEM MODEL}

The block diagram of our low-density parity-check (LDPC)coded SEFDM-IM transceiver is depicted in Fig. 1. At the transmitter side, $N_{b}$ information bits $\mathbf{b}=\left[b_{0}, \cdots, b_{N_{b}-1}\right]^{T}$ are encoded and then $N_{c}$ coded bits $\mathbf{c}=\left[c_{0}, \cdots, c_{N_{c}-1}\right]^{T}$ are equally partitioned into $G$ groups, each containing $P=N_{c} / G$ bits, i.e., $\mathbf{c}_{g}=\left[c_{g, 0}, \cdots, c_{g, p}, \cdots, c_{g, P-1}\right]^{T}$, where $c_{g, p}$ is equal to the $[(g-1) P+p]$-th element of $\mathbf{c}$. Each group of $P$ coded bits is mapped to an SEFDM-IM subblock of length $N$, where $N=N_{s} / G$ and $N_{s}$ is the number of SEFDM-IM subcarriers. For each SEFDM-IM subblock, only $K$ out of $N$ subcarriers are activated to transmit $M$-ary symbols, where the first $P_{1}=\log _{2}\left\lfloor\left(\begin{array}{l}N \\ K\end{array}\right)\right\rfloor$ of $P$ bits are used for determining $K$ indices of the activated subcarriers, while the remaining $P_{2}=K \log _{2} M$ bits are mapped onto the constellation points $\mathcal{S}$. The activated indices and modulated $M$-ary symbols of the $g$-th subblock are denoted as $\mathbf{I}_{g}=\left\{I_{g, 1}, I_{g, 2}, \ldots, I_{g, K}\right\}, I_{g, k} \in\{1, \ldots, N\}$ and 


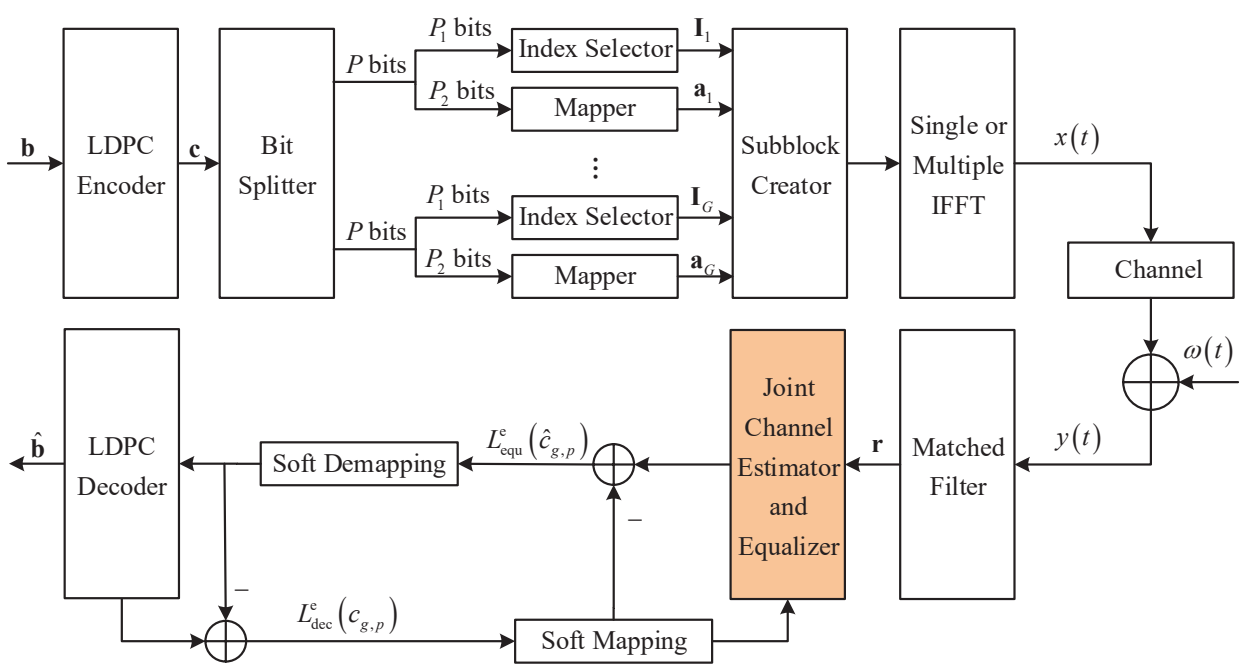

Fig. 1. Block diagram of an LDPC-coded SEFDM-IM system.

$\mathbf{a}_{g}=\left[a_{g, 1}, a_{g, 2}, \ldots, a_{g, K}\right]^{T}, a_{g, k} \in \mathcal{S}$. The power of the signal constellation is normalized to unit average power, i.e., $\mathbb{E}\left\{\mathbf{a}_{g}^{H} \mathbf{a}_{g}\right\}=K$. The vector of the modulated SEFDM symbols in the $g$-th subblock is $\mathbf{x}_{g}=\left[x_{g, 1}, \cdots, x_{g, N}\right]^{T}$, where $x_{g, n}=a_{g, k}$ for $n=I_{g, k} \in \mathbf{I}_{g}$ and $x_{g, n}=0$ for $n \notin \mathbf{I}_{g}$. Then, $G$ subblocks are concatenated to generate the $N_{s}$ transmitted SEFDM-IM symbols, i.e., $\mathbf{x}=\left[\mathbf{x}_{1}^{T}, \cdots, \mathbf{x}_{G}^{T}\right]^{T}$. To enhance the spectral efficiency, the complex-valued symbols $\mathbf{x}$ are mapped to $N_{s}$ non-orthogonal subcarriers relying on the bandwidth packing factor of $\alpha=\Delta f_{s} T_{s}$, where $\Delta f_{s}$ is the spacing of subcarriers and $T_{s}$ is the SEFDM-IM signal duration. The inverse discrete Fourier transform (IDFT) can be used for modulation in our SEFDM-IM systems [32]. Consequently, the equivalent baseband signal is expressed as

$$
x(t)=\sqrt{\frac{N}{K T_{s}}} \sum_{n=0}^{N_{s}-1} x_{n} e^{\frac{j 2 \pi \alpha n t}{T_{s}}},
$$

where $x_{n}$ is the $n$-th modulated symbol, and $\sqrt{N / K}$ is the signal power normalization factor.

Without loss of generality, we assume that the length of cyclic prefix is sufficiently for intersymbol interference (ISI)free transmission over an $L$-tap frequency-selective fading channel. The received SEFDM-IM signal is given by

$$
y(t)=\sum_{l=0}^{L-1} \bar{h}_{l} x\left(t-\tau_{l}\right)+\omega(t),
$$

where $\bar{h}_{l}$ and $\tau_{l}$ are the CIR and the delay of the $l$-th path, respectively, and $\omega(t)$ is the additive white Gaussian noise (AWGN) process with zero mean and variance $\sigma_{\omega}^{2}$.

To support reconfigurable architectures and facilitate compatibility with the receivers of the existing systems, instead of introducing additional orthonormalization operation, we adopt a non-orthogonal matched filtering [33]. The $k$-th output of the non-orthogonal matched filter is

$$
\begin{aligned}
r_{k} & =\sqrt{\frac{K}{N T_{s}}} \int_{0}^{T_{s}} y(t) e^{-\frac{j 2 \pi \alpha k t}{T_{s}}} d t \\
& =\frac{1}{T_{s}} \sum_{n=0}^{N_{s}-1} x_{n} \sum_{l=0}^{L-1} \bar{h}_{l} e^{-\frac{j 2 \pi \alpha n \tau_{l}}{T_{s}}} \int_{0}^{T_{s}} e^{-\frac{j 2 \pi \alpha(k-n) t}{T_{s}}} d t+\omega_{k} \\
& =\sum_{n=0}^{N_{s}-1} \phi_{k, n} h_{n} x_{n}+\omega_{k}
\end{aligned}
$$

where $h_{n}=\sum_{l=0}^{L-1} \bar{h}_{l} e^{-\frac{j 2 \pi \alpha n \tau_{l}}{T_{s}}}$ is the $n$-th channel tap in the frequency domain, $\omega_{k}=\sqrt{\frac{K}{N T_{s}}} \int_{0}^{T_{s}} \omega(t) e^{-\frac{j 2 \pi \alpha k t}{T_{s}}} d t$ is the inherent colored noise having the complex-valued autocorrelation matrix $\mathbb{E}\left\{\boldsymbol{\omega} \boldsymbol{\omega}^{H}\right\}=K \sigma_{\omega}^{2} / N \boldsymbol{\Phi}, \boldsymbol{\omega}=\left[\omega_{0}, \cdots, \omega_{N-1}\right]^{T}$, and $\boldsymbol{\Phi}$ denotes the interference matrix with elements $\phi_{k, n}=$ $e^{-j \pi \alpha(k-n)} \operatorname{sinc}[\alpha(k-n)]$.

\section{GMP-EP EQUALIZATION WITH KNOWN CSI}

In this section, we focus our attention on the joint interference mitigation and detection of SEFDM-IM systems under the assumption of perfectly known CSI. By introducing the extended constellation containing deactivated subcarriers and reformulating the SEFDM-IM equalization design via a linear state-space model, we construct the factor graph having two subgraphs corresponding to the symbol-based interference mitigation and the colored noise approximation. Besides, discrete input messages are parameterized by a Gaussian form based on EP, hence all messages on factor graph are derived using GMP rules. This work independently solves the known interference elimination problem of SEFDM-IM systems, whilst additionally providing a valuable research basis for joint channel estimation and equalization techniques for SEFDM-IM signaling in Section IV.

\section{A. Factor Graph Model for SEFDM-IM Systems Having Known CSI}

Due to the inter-dependence of the SEFDM-IM subcarriers in a subblock, conventional receivers typically rely on a multistage demodulation strategy [30], i.e., the interferences 
are eliminated in the first stage and then ML detection is employed for recovering the index bits and the symbol bits in the second stage. To develop a low-complexity joint interference mitigation and IM detection algorithm for SEFDM-IM signaling, we intentionally introduce an extended constellation for incorporating the deactivated subcarriers of zeros. Accordingly, activated pattern constraints can be included as the $a$ priori probability distribution of each transmitted symbol.

Owing to the fact that the ICI introduced by packing the non-orthogonal subcarriers decreases as the subcarrier spacing increases, only the interferences from the immediately adjacent subcarriers are considered for reducing the complexity. Accordingly, the received signal in (3) is rewritten as

$$
r_{k}=\phi_{k}^{T} \mathbf{s}_{k}+\omega_{k}
$$

where $\phi_{k}=\left[\phi_{k, k-L_{t}}, \ldots, \phi_{k, k}, \ldots, \phi_{k, k+L_{t}}\right]^{T}, \mathbf{s}_{k}=$ $\left[s_{k-L_{t}}, \ldots, s_{k}, \ldots, s_{k+L_{t}}\right]^{T}$ with $s_{k}=h_{k} x_{k}, x_{k}$ belongs to the extended constellation $\{0, \mathcal{S}\}$ and $L_{t}$ is the length of the truncated interferences. The variables $\mathbf{s}_{k-1}$ and $\mathbf{s}_{k}$ satisfy the following linear state transition model of

$$
\mathbf{s}_{k}=\boldsymbol{\Xi}_{1} \mathbf{s}_{k-1}+\zeta_{1} s_{k+L_{t}},
$$

where $\boldsymbol{\Xi}_{1}=\left[\mathbf{0}_{L_{s}-1}^{T}, \mathbf{I}_{L_{s}-1} ; \mathbf{0}_{L_{s}}\right], \boldsymbol{\zeta}_{1}=\left[\mathbf{0}_{L_{s}-1}, 1\right]^{T}$ with $L_{s}=$ $2 L_{t}+1$.

In contrast to the colored noise in the time domain (TD), the autocorrelation matrix of the frequency-domain (FD) colored noise is a complex-valued Toeplitz matrix. We employ the $\bar{P}$ th order CAR model [34] for characterizing the colored noise as

$$
\xi_{k}=\sum_{\bar{p}=1}^{\bar{P}} \lambda_{\bar{p}} \xi_{k-\bar{p}}+\tilde{\xi}_{k}=\boldsymbol{\lambda}^{T} \boldsymbol{\xi}_{k-1}+\tilde{\xi}_{k},
$$

where $\boldsymbol{\lambda}=\left[\lambda_{1}, \cdots, \lambda_{\bar{P}}\right]^{T}$ is the complex-valued coefficient vector, $\boldsymbol{\xi}_{k-1}=\left[\xi_{k-1}, \cdots, \xi_{k-\bar{P}}\right]^{T}$ is defined as the state vector, while $\tilde{\xi}_{k}$ is a complex-valued AWGN sample with zero mean and variance $\sigma_{\tilde{\xi}}^{2}=\phi_{0,0}-\bar{\phi}^{H} \boldsymbol{\lambda}$. By minimizing the mean squared error, i.e., $\mathbb{E}\left\{\left|\tilde{\xi}_{k}\right|^{2}\right\}$, the optimal CAR coefficients are given by $\boldsymbol{\lambda}=\mathbf{R}^{-1}(\bar{P}) \bar{\phi}$, where $\mathbf{R}(\bar{P})=$ $\frac{K \sigma_{\omega}^{2}}{N} \cdot\left[\phi_{0,0}, \phi_{0,1}, \ldots, \phi_{0, \bar{P}-1} ; \cdots ; \phi_{\bar{P}-1,0}, \phi_{\bar{P}-1,1}, \ldots, \phi_{0,0}\right]$ and $\bar{\phi}=\left[\phi_{0,1}, \ldots, \phi_{0, \bar{P}}\right]^{T}$. Then (4) can be rewritten as

$$
r_{k}=\phi_{k}^{T} \mathbf{s}_{k}+\xi_{k}
$$

where $\xi_{k}=\boldsymbol{\zeta}_{2}^{T} \boldsymbol{\xi}_{k}$ with $\boldsymbol{\zeta}_{2}=\left[1, \mathbf{0}_{\bar{P}-1}^{T}\right]^{T}$. The linear transition constraint between $\boldsymbol{\xi}_{k}$ and $\boldsymbol{\xi}_{k-1}$ is

$$
\boldsymbol{\xi}_{k}=\boldsymbol{\Xi}_{2} \boldsymbol{\xi}_{k-1}+\boldsymbol{\zeta}_{2} \tilde{\xi}_{k}
$$

where $\boldsymbol{\Xi}_{2}=\left[\boldsymbol{\lambda}^{T} ; \mathbf{I}_{\bar{P}-1}, \mathbf{0}_{\bar{P}-1}\right]$.

Based on (5)-(8), we construct the factor graph of our SEFDM-IM system with known CSI, as shown in Fig. 2. Subgraph 1 and Subgraph 2 correspond to the symbol-wise interference mitigation and the colored noise approximation, respectively. The edges denote variables and the factor nodes represent local functions. The natural schedule for the message computations on factor graph consists of two independent recursions, namely, forward message passing along the direction of the arrow and backward message passing opposite the direction of the arrow. In the following, we will derive the messages on the factor graph in details.

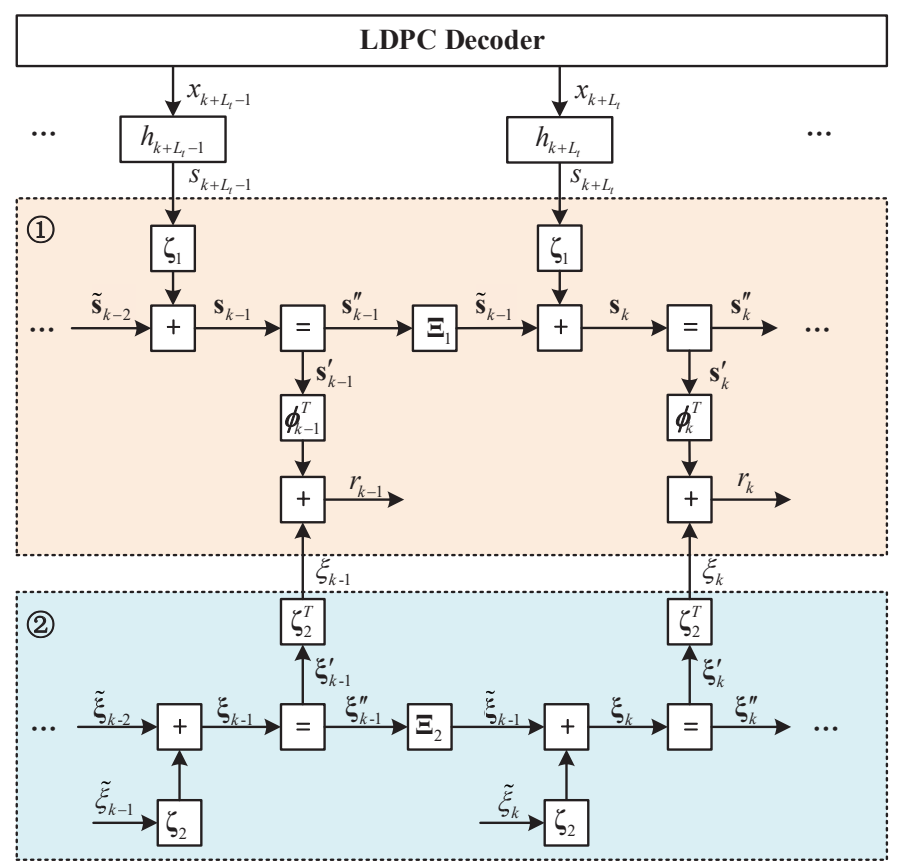

Fig. 2. Factor graph for equalization of SEFDM-IM signaling with known CSI.

\section{B. Hybrid GMP-EP Equalization}

We employ GMP to update messages on FFG [35], [36] in Fig. 2. The forward and backward messages are characterized by the mean vector $\overrightarrow{\mathbf{m}}$ and the covariance matrix $\overrightarrow{\mathbf{V}}$ or the transformed mean vector $\overleftarrow{\mathbf{W}} \overleftarrow{\mathbf{m}}$ and the weight matrix $\overleftarrow{\mathbf{W}}=$ $\overrightarrow{\mathbf{V}^{-1}}$, respectively.

- Gaussian Message Updating for Interference Mitigation

Assuming that the mean vector $\overrightarrow{\mathbf{m}}_{\mathbf{s}_{k-1}}^{\left(i_{t}\right)}$ and covariance matrix $\overrightarrow{\mathbf{V}}_{\mathbf{s}_{k-1}}^{\left(i_{t}\right)}$ at the $i_{t}$-th inner iteration are available, the forward messages of the variables $\mathbf{s}_{k-1}^{\prime \prime}$ are given by

$$
\begin{aligned}
& \overrightarrow{\mathbf{m}}_{\mathbf{s}_{k-1}^{\prime \prime}}^{\left(i_{t}\right)}=\overrightarrow{\mathbf{m}}_{\mathbf{s}_{k-1}}^{\left(i_{t}\right)}+\frac{\overrightarrow{\mathbf{V}}_{\mathbf{s}_{k-1}}^{\left(i_{t}\right)}\left(\boldsymbol{\phi}_{k-1}^{T}\right)^{H}\left(r_{k-1}-\boldsymbol{\phi}_{k-1}^{T} \overrightarrow{\mathbf{m}}_{\mathbf{s}_{k-1}}^{\left(i_{t}\right)}\right)}{\vec{V}_{\xi_{k-1}}^{\left(i_{t}-1\right)}+\boldsymbol{\phi}_{k-1}^{T} \overrightarrow{\mathbf{V}}_{\mathbf{s}_{k-1}}^{\left(i_{t}\right)}\left(\boldsymbol{\phi}_{k-1}^{T}\right)^{H}}, \\
& \overrightarrow{\mathbf{V}}_{\mathbf{s}_{k-1}^{\prime \prime}}^{\left(i_{t}\right)}=\overrightarrow{\mathbf{V}}_{\mathbf{s}_{k-1}}^{\left(i_{t}\right)}-\frac{\overrightarrow{\mathbf{V}}_{\mathbf{s}_{k-1}}^{\left(i_{i}\right)}\left(\boldsymbol{\phi}_{k-1}^{T}\right)^{H} \boldsymbol{\phi}_{k-1}^{T} \overrightarrow{\mathbf{V}}_{\mathbf{s}_{k-1}}^{\left(i_{t}\right)}}{\vec{V}_{\xi_{k-1}}^{\left(i_{t}-1\right)}+\boldsymbol{\phi}_{k-1}^{T} \overrightarrow{\mathbf{V}}_{\mathbf{s}_{k-1}}^{\left(i_{t}\right)}\left(\boldsymbol{\phi}_{k-1}^{T}\right)^{H}},
\end{aligned}
$$

where $r_{k-1}$ is the $k$-th sample of the received SEFDM-IM signal and $\vec{V}_{\xi_{k-1}}^{\left(i_{t}-1\right)}$ is the forward variance of the approximate noise $\xi_{k-1}$ in the $\left(i_{t}-1\right)$-th inner iteration. Based on the forward updating rules of the matrix multiplication node, the forward mean vector and covariance matrix of $\tilde{\mathbf{s}}_{k-1}$ are obtained, respectively, as

$$
\begin{aligned}
& \overrightarrow{\mathbf{m}}_{\tilde{\mathbf{s}}_{k-1}}^{\left(i_{t}\right)}=\boldsymbol{\Xi}_{1} \overrightarrow{\mathbf{m}}_{\mathbf{s}_{k-1}^{\prime \prime}}^{\left(i_{t}\right)}, \\
& \overrightarrow{\mathbf{V}}_{\tilde{\mathbf{s}}_{k-1}}^{\left(i_{t}\right)}=\boldsymbol{\Xi}_{1} \overrightarrow{\mathbf{V}}_{\mathbf{s}_{k-1}^{\prime \prime}}^{\left(i_{t}\right)} \boldsymbol{\Xi}_{1}^{H} .
\end{aligned}
$$

Based on (9)-(12), the forward messages of $\mathbf{s}_{k}$ can be updated as

$$
\begin{aligned}
& \overrightarrow{\mathbf{m}}_{\mathbf{s}_{k}}^{\left(i_{t}\right)}=\overrightarrow{\mathbf{m}}_{\tilde{\mathbf{s}}_{k-1}}^{\left(i_{t}\right)}+h_{k+L_{t}} \vec{m}_{x_{k+L_{t}}}^{\left(i_{t}-1\right)} \boldsymbol{\zeta}_{1}, \\
& \overrightarrow{\mathbf{V}}_{\mathbf{s}_{k}}^{\left(i_{t}\right)}=\overrightarrow{\mathbf{V}}_{\tilde{\mathbf{s}}_{k-1}}^{\left(i_{t}\right)}+\left|h_{k+L_{t}}\right|^{2} \vec{V}_{x_{k+L_{t}}}^{\left(i_{t}-1\right)} \boldsymbol{\zeta}_{1} \boldsymbol{\zeta}_{1}^{H},
\end{aligned}
$$


where $h_{k+L_{t}}$ is the known channel state information on the $\left(k+L_{t}\right)$-th subcarrier, $\vec{m}_{x_{k+L_{t}}}^{\left(i_{t}-1\right)}$ and $\vec{V}_{x_{k+L_{t}}}^{\left(i_{t}-1\right)}$ are the a priori mean and variance of the $\left(k+L_{t}\right)$-th transmitted symbol, which will be elaborated on later.

Similar to the derivations of forward messages, assuming that the weight matrix $\overleftarrow{\mathbf{W}}_{\mathbf{s}_{k}}^{\left(i_{t}\right)}$ and the transformed mean vector $\overleftarrow{\mathbf{W}}_{\mathbf{s}_{k}}^{\left(i_{t}\right)} \overleftarrow{\mathbf{m}}_{\mathbf{s}_{k}}^{\left(i_{t}\right)}$ are available, we are able to calculate the backward messages of $\tilde{\mathbf{s}}_{k-1}$ as

$$
\begin{aligned}
& \overleftarrow{\mathbf{W}}_{\tilde{\mathbf{s}}_{k-1}}^{\left(i_{t}\right)}=\overleftarrow{\mathbf{W}}_{\mathbf{s}_{k}}^{\left(i_{t}\right)}-\frac{\left|h_{k+L_{t}}\right|^{2} \vec{V}_{x_{k+L_{t}}}^{\left(i_{t}-1\right)} \overleftarrow{\mathbf{W}}_{\mathbf{s}_{k}}^{\left(i_{t}\right)} \boldsymbol{\zeta}_{1} \boldsymbol{\zeta}_{1}^{H} \overleftarrow{\mathbf{W}}_{\mathbf{s}_{k}}^{\left(i_{t}\right)}}{1+\left|h_{k+L_{t}}\right|^{2} \vec{V}_{x_{k+L_{t}}}^{\left(i_{t}-1\right)} \boldsymbol{\zeta}_{1}^{H} \overleftarrow{\mathbf{W}}_{\mathbf{s}_{k}}^{\left(i_{t}\right)} \boldsymbol{\zeta}_{1}} \\
& \overleftarrow{\mathbf{W}}_{\tilde{\mathbf{s}}_{k-1}}^{\left(i_{t}\right)} \overleftarrow{\mathbf{m}}_{\tilde{\mathbf{s}}_{k-1}}^{\left(i_{t}\right)}=\left(\mathbf{I}-\frac{\left|h_{k+L_{t}}\right|^{2} \vec{V}_{x_{k+L_{t}}}^{\left(i_{t}-1\right)} \overleftarrow{\mathbf{W}}_{\mathbf{s}_{k}}^{\left(i_{t}\right)} \boldsymbol{\zeta}_{1} \boldsymbol{\zeta}_{1}^{H}}{1+\left|h_{k+L_{t}}\right|^{2} \vec{V}_{x_{k+L_{t}}}^{\left(i_{t}-1\right)} \boldsymbol{\zeta}_{1}^{H} \overleftarrow{\mathbf{W}}_{\mathbf{s}_{k}}^{\left(i_{t}\right)} \boldsymbol{\zeta}_{1}}\right) \\
& \times\left(\overleftarrow{\mathbf{W}}_{\mathbf{s}_{k}}^{\left(i_{t}\right)} \overleftarrow{\mathbf{m}}_{\mathbf{s}_{k}}^{\left(i_{t}\right)}-h_{k+L_{t}} \vec{m}_{x_{k+L_{t}}}^{\left(i_{t}-1\right)} \overleftarrow{\mathbf{W}}_{\mathbf{s}_{k}}^{\left(i_{t}\right)} \boldsymbol{\zeta}_{1}\right)
\end{aligned}
$$

Then, the backward messages of $\mathbf{s}_{k-1}^{\prime \prime}$ are given by

$$
\begin{gathered}
\overleftarrow{\mathbf{W}}_{\mathbf{s}_{k-1}^{\prime \prime}}^{\left(i_{t}\right)}=\boldsymbol{\Xi}_{1}^{H} \overleftarrow{\mathbf{W}}_{\tilde{\mathbf{s}}_{k-1}}^{\left(i_{t}\right)} \boldsymbol{\Xi}_{1} \\
\overleftarrow{\mathbf{W}}_{\mathbf{s}_{k-1}^{\prime \prime}}^{\left(i_{t}\right)} \overleftarrow{\mathbf{m}}_{\mathbf{s}_{k-1}^{\prime \prime}}^{\left(i_{t}\right)}=\boldsymbol{\Xi}_{1}^{H} \overleftarrow{\mathbf{W}}_{\tilde{\mathbf{s}}_{k-1}}^{\left(i_{t}\right)} \overleftarrow{\mathbf{m}}_{\tilde{\mathbf{s}}_{k-1}}^{\left(i_{i}\right)}
\end{gathered}
$$

Based on (15)-(18), the backward messages of the state variables $\mathbf{s}_{k-1}$ can be updated as

$$
\begin{gathered}
\overleftarrow{\mathbf{W}}_{\mathbf{s}_{k-1}}^{\left(i_{t}\right)}=\overleftarrow{\mathbf{W}}_{\mathbf{s}_{k-1}^{\prime \prime}}^{\left(i_{t}\right)}+\frac{\left(\phi_{k-1}^{T}\right)^{H} \boldsymbol{\phi}_{k-1}^{T}}{\vec{V}_{\xi_{k-1}}^{\left(i_{t}-1\right)}} \\
\overleftarrow{\mathbf{W}}_{\mathbf{s}_{k-1}}^{\left(i_{t}\right)} \overleftarrow{\mathbf{m}}_{\mathbf{s}_{k-1}}^{\left(i_{t}\right)}=\overleftarrow{\mathbf{W}}_{\mathbf{s}_{k-1}^{\prime \prime}}^{\left(i_{t}\right)} \overleftarrow{\mathbf{m}}_{\mathbf{s}_{k-1}^{\prime \prime}}^{\left(i_{t}\right)}+\frac{\left(\phi_{k-1}^{T}\right)^{H} r_{k-1}}{\vec{V}_{\xi_{k-1}}^{\left(i_{t}-1\right)}}
\end{gathered}
$$

where $\vec{V}_{\xi_{k-1}}^{\left(i_{t}-1\right)}$ is updated on Subgraph 2 in the previous iteration. From (14) and (20), we have the a posteriori variance of the state vector $\mathbf{s}_{k}$ as

$$
\mathbf{V}_{\mathbf{s}_{k}}^{\left(i_{t}\right)}=\left(\left(\overrightarrow{\mathbf{V}}_{\mathbf{s}_{k}}^{\left(i_{t}\right)}\right)^{-1}+\overleftarrow{\mathbf{W}}_{\mathbf{s}_{k}}^{\left(i_{t}\right)}\right)^{-1}
$$

In our turbo receiver, soft extrinsic information is exchanged between the channel decoder and the SEFDM-IM equalizer, as shown in Fig. 1. Hence, the a priori messages of the transmitted symbols $\mathbf{x}=\left[x_{0}, \cdots, x_{N-1}\right]^{T}$ in (13)-(16) are obtained using the extrinsic LLRs gleaned from the output of the channel decoder in the outer iterations. Note that the inner iterations within the equalizer are embedded into the outer iterations between the equalizer and the channel decoder. For the $i_{o}$-th outer iteration, the extrinsic LLRs of the channel decoder are fixed when the inner iterations are performed. Assuming that the $p$-th extrinsic LLR of the channel decoder output in the $g$-th SEFDM-IM subblock is $L_{\mathrm{dec}}^{\mathrm{e},\left(i_{o}\right)}\left(c_{g, p}\right)$, the a priori probability of the coded bit is $P^{\left(i_{o}\right)}\left(c_{g, p}\right)=\frac{1}{2}\left[1+(-1)^{c_{g, p}} \tanh \left(\frac{1}{2} L_{\mathrm{dec}}^{\mathrm{e},\left(i_{o}\right)}\left(c_{g, p}\right)\right)\right]$, where $c_{g, p} \in\{0,1\}, p=0, \cdots, P-1$.

As mentioned before, each transmitted SEFDM-IM symbol belongs to the extended constellation $\left\{\mathcal{S}_{1}, \cdots, \mathcal{S}_{M}, 0\right\}$. The $a$ priori distribution of the $n$-th transmitted symbol has to be calculated based on the specific subcarrier activation pattern constraints and on the classic $M$-ary mapping rules using the a priori probabilities of the whole coded bits in the subblock. Let us assume that $K$ indices of the $m_{1}$-th activated pattern $\mathbf{I}_{m_{1}}=\left\{I_{m_{1}, 1}, \ldots, I_{m_{1}, K}\right\}$ correspond to the coded index modulation bits $\mathbf{c}_{m_{1}}=\left[c_{m_{1}, 1}, \cdots, c_{m_{1}, P_{1}}\right]^{T}, m_{1}=$ $1, \cdots, 2^{P_{1}}$ and the $m_{2}$-th constellation candidate $\mathbf{a}_{m_{2}}=$ $\left[a_{m_{2}, 1}, \ldots, a_{m_{2}, K}\right]$ corresponds to the coded modulated symbol bits $\mathbf{c}_{\dot{m}_{2}}=\left[c_{m_{2}, 1}, \cdots, c_{m_{2}, P_{2}}\right]^{T}, m_{2}=1, \cdots, 2^{P_{2}}$. The a priori probability of the $n$-th modulated symbol in the $g$-th subblock is expressed as

$$
\begin{aligned}
& P_{g, n, 0}^{\left(i_{o}\right)}=\sum_{m_{1}, n \notin \mathbf{I}_{m_{1}}}\left[\prod_{p_{1}=1}^{P_{1}} P^{\left(i_{o}\right)}\left(c_{g, p_{1}}=c_{m_{1}, p_{1}}\right)\right] \\
& P_{g, n, m}^{\left(i_{o}\right)}=\sum_{m_{1}, n \in \mathbf{I}_{m_{1}}}\left[\prod_{p_{1}=1}^{P_{1}} P^{\left(i_{o}\right)}\left(c_{g, p_{1}}=c_{m_{1}, p_{1}}\right) \prod_{p_{2}=P_{1}+1}^{P} P^{\left(i_{o}\right)}\left(c_{g, p_{2}}=c_{m_{2}, p_{2}-P_{1}}\right)\right]
\end{aligned}
$$

where $c_{g, p_{1}}$ denotes the index bits, while $c_{g, p_{2}}$ represents the classic modulated symbol bits.

Nevertheless, the discrete distribution of the incoming message imposes exponentially increased complexity. To circumvent this, we resort to approximating the distribution by a Gaussian form. Second-order moment matching is a fairly straightforward method, but suffers from an excessive approximation error. By contrast, EP is an efficient technique of approximating a posteriori beliefs that belong to the exponential distribution families [37], [38]. The approximated Gaussian distribution is derived via minimizing the KLD between the true marginal distribution $b^{\left(i_{t}\right)}\left(x_{k}\right)$ and the trial distribution $b_{G}^{\left(i_{t}\right)}\left(x_{k}\right)$, i.e.,

$$
b_{G}^{\left(i_{t}\right)}\left(x_{k}\right)=\arg \min _{b_{G}^{\left(i_{t}\right)}\left(x_{k}\right)} D_{\mathrm{KL}}\left(b^{\left(i_{t}\right)}\left(x_{k}\right) \| b_{G}^{\left(i_{t}\right)}\left(x_{k}\right)\right),
$$

where the a priori probability of the transmitted symbol $x_{k}$ depends on both $P_{g, n, 0}$ and on $P_{g, n, m}$ for $k=(g-1) N+n-1$. The projection of a univariate distribution onto the Gaussian distribution in (24) is equivalent to matching the moments of the discrete a posteriori distribution and the Gaussian distribution [39]. Having the a priori distributions and the outgoing Gaussian messages at the $i_{t}$-th inner iteration, the first-order and second-order moment of the a posteriori distribution $b^{\left(i_{t}\right)}\left(x_{k}\right)$ are given by

$$
\begin{aligned}
m_{x_{k}}^{\left(i_{t}\right)}= & \frac{1}{\varepsilon_{0} \pi \overleftarrow{V}\left(i_{x_{k}}\right)} \sum_{m_{2}=1}^{M} \chi_{m_{2}} P_{g, n, m_{2}}^{\left(i_{o}\right)} \exp \left(-\frac{\left|\chi_{m_{2}}-\overleftarrow{m}_{x_{k}}^{\left(i_{t}\right)}\right|^{2}}{\overleftarrow{V}\left(i_{x_{k}}-1\right)}\right) \\
V_{x_{k}}^{\left(i_{t}\right)}= & \frac{\left|m_{x_{k}}^{\left(i_{t}\right)}\right|^{2} P_{g, n, 0}^{\left(i_{o}\right)}}{\varepsilon_{0} \pi \overleftarrow{V}_{x_{k}}^{\left(i_{t}\right)}} \exp \left(-\frac{\left|\overleftarrow{m}_{x_{k}}^{\left(i_{t}\right)}\right|^{2}}{\overleftarrow{V}_{x_{k}}^{\left(i_{t}\right)}}\right)+\frac{\sum_{m_{2}=1}^{M}\left|\chi_{m_{2}}-m_{x_{k}}^{\left(i_{t}\right)}\right|^{2}}{\varepsilon_{0} \pi \overleftarrow{V}_{x_{k}}^{\left(i_{t}\right)}} \\
& \times P_{g, n, m_{2}}^{\left(i_{o}\right)} \exp \left(-\frac{\left|\chi_{m_{2}}-\overleftarrow{m}_{x_{k}}^{\left(i_{t}\right)}\right|^{2}}{\overleftarrow{V}_{x_{k}}^{\left(i_{t}\right)}}\right)-\left|m_{x_{k}}^{\left(i_{t}\right)}\right|^{2}
\end{aligned}
$$

where $\overleftarrow{m}_{x_{k}}^{\left(i_{t}\right)}$ and $\overleftarrow{V}_{x_{k}}^{\left(i_{t}\right)}$ are the mean and variance of the outgoing message, while $\varepsilon_{0}$ is the normalization factor of the 
a posteriori distribution. Based on (25) and (26), the forward messages arriving from the channel decoder are

$$
\begin{aligned}
\vec{m}_{x_{k}}^{\left(i_{t}\right)} & =\frac{m_{x_{k}}^{\left(i_{t}\right)} \overleftarrow{V}_{x_{k}}^{\left(i_{t}\right)}-\overleftarrow{m}_{x_{k}}^{\left(i_{t}\right)} V_{x_{k}}^{\left(i_{t}\right)}}{\overleftarrow{V}_{x_{k}}^{\left(i_{t}\right)}-V_{x_{k}}^{\left(i_{t}\right)}} \\
\vec{V}_{x_{k}}^{\left(i_{t}\right)} & =\frac{\overleftarrow{V}_{x_{k}}^{\left(i_{t}\right)} V_{x_{k}}^{\left(i_{t}\right)}}{\overleftarrow{V}_{x_{k}}^{\left(i_{t}\right)}-V_{x_{k}}^{\left(i_{t}\right)}}
\end{aligned}
$$

For expressing the extrinsic LLR gleaned from the equalizer for the processing of the channel decoder, we introduce the auxiliary quantity [35] of the state $\mathbf{s}_{k}$ as

$$
\tilde{\mathbf{W}}_{\mathbf{s}_{k}}^{\left(i_{t}\right)}=\left(\overrightarrow{\mathbf{V}}_{\mathbf{s}_{k}}^{\left(i_{t}\right)}+\overleftarrow{\mathbf{V}}_{\mathbf{s}_{k}}^{\left(i_{t}\right)}\right)^{-1}
$$

Thus, given the CSI, the backward mean and variance of the transmitted symbol at the $i_{t}$-th inner iteration are formulated as

$$
\begin{aligned}
& \overleftarrow{V}_{x_{k+L_{t}}}^{\left(i_{t}\right)}=\frac{1-\vec{V}_{s_{k+L_{t}}}^{\left(i_{t}-1\right)}}{\left|H_{k+L_{t}}\right|^{2} \boldsymbol{\zeta}_{1}^{H} \tilde{\mathbf{W}}_{\mathbf{s}_{k}}^{\left(i_{t}\right)} \boldsymbol{\zeta}_{1}}, \\
& \overleftarrow{m}_{x_{k+L_{t}}}^{\left(i_{t}\right)}=\frac{H_{k+L_{t}}^{*} \boldsymbol{\zeta}_{1}^{H} \tilde{\mathbf{W}}_{\mathbf{s}_{k}}^{\left(i_{t}\right)}\left(\overleftarrow{\mathbf{m}}_{\mathbf{s}_{k}}^{\left(i_{t}\right)}-\overleftarrow{\mathbf{m}}_{\tilde{\mathbf{s}}_{k}}^{\left(i_{t}\right)}\right)}{\left|H_{k+L_{t}}\right|^{2} \boldsymbol{\zeta}_{1}^{H} \tilde{\mathbf{W}}_{\mathbf{s}_{k}}^{\left(i_{t}\right)} \boldsymbol{\zeta}_{1}}
\end{aligned}
$$

Then, the extrinsic LLR output by the equalizer per subblock can be calculated by the corresponding expressions in [40], [41].

- Gaussian Message Updating for Colored Noise Approximation

The forward and backward messages on Subgraph 2 are calculated based on GMP rules. Assuming that the covariance matrix $\overrightarrow{\mathbf{V}}_{\boldsymbol{\xi}_{k-1}}^{\left(i_{t}\right)}$ is available, the forward variance vector of $\boldsymbol{\xi}_{k-1}^{\prime \prime}$ is expressed as

$$
\overrightarrow{\mathbf{V}}_{\boldsymbol{\xi}_{k-1}^{\prime \prime}}^{\left(i_{t}\right)}=\overrightarrow{\mathbf{V}}_{\boldsymbol{\xi}_{k-1}}^{\left(i_{t}\right)}-\frac{\overrightarrow{\mathbf{V}}_{\boldsymbol{\xi}_{k-1}}^{\left(i_{t}\right)}\left(\boldsymbol{\zeta}_{2}^{T}\right)^{H} \boldsymbol{\zeta}_{2}^{T} \overrightarrow{\mathbf{V}}_{\boldsymbol{\xi}_{k-1}}^{\left(i_{t}\right)}}{\overleftarrow{V}_{\xi_{k}}+\boldsymbol{\zeta}_{2}^{T} \overrightarrow{\mathbf{V}}_{\boldsymbol{\xi}_{k-1}}\left(\boldsymbol{\zeta}_{2}^{T}\right)^{H}}
$$

Similar to (12) and (14), the forward covariance matrix $\overrightarrow{\mathbf{V}}_{\xi_{k}}^{\left(i_{t}\right)}$ is represented as

$$
\overrightarrow{\mathbf{V}}_{\boldsymbol{\xi}_{k}}^{\left(i_{t}\right)}=\boldsymbol{\Xi}_{2} \overrightarrow{\mathbf{V}}_{\boldsymbol{\xi}_{k-1}^{\prime \prime}}^{\left(i_{t}\right)} \boldsymbol{\Xi}_{2}^{H}+N_{\tilde{\xi}} \boldsymbol{\zeta}_{2} \boldsymbol{\zeta}_{2}^{H}
$$
have

Assuming that the weight matrix $\overleftarrow{\mathbf{W}}_{\boldsymbol{\xi}_{k}}^{\left(i_{t}\right)}$ is available, we

$$
\overleftarrow{\mathbf{W}}_{\tilde{\boldsymbol{\xi}}_{k-1}}^{\left(i_{t}\right)}=\overleftarrow{\mathbf{W}}_{\boldsymbol{\xi}_{k}}^{\left(i_{t}\right)}-\frac{\sigma_{\tilde{\xi}}^{2} \overleftarrow{\mathbf{W}}_{\boldsymbol{\xi}_{k}}^{\left(i_{t}\right)} \zeta_{2} \boldsymbol{\zeta}_{2}^{H} \overleftarrow{\mathbf{W}}_{\boldsymbol{\xi}_{k}}^{\left(i_{t}\right)}}{1+\sigma_{\tilde{\xi}}^{2} \boldsymbol{\zeta}_{2}^{H} \overleftarrow{\mathbf{W}}_{\boldsymbol{\xi}_{k}}^{\left(i_{t}\right)} \boldsymbol{\zeta}_{2}}
$$

Then, the backward weight matrix $\overleftarrow{\mathbf{W}}_{\boldsymbol{\xi}_{k-1}}^{\left(i_{t}\right)}$ is updated as

$$
\overleftarrow{\mathbf{W}}_{\boldsymbol{\xi}_{k-1}}^{\left(i_{t}\right)}=\boldsymbol{\Xi}_{2}^{H} \overleftarrow{\mathbf{W}}_{\tilde{\boldsymbol{\xi}}_{k-1}}^{\left(i_{t}\right)} \boldsymbol{\Xi}_{2}+\frac{\boldsymbol{\zeta}_{2}^{H} \zeta_{2}}{\overleftarrow{V}_{\xi_{k}}^{\left(i_{t}-1\right)}}
$$

where $\overleftarrow{V}_{\xi_{k}}^{\left(i_{t}\right)}$ is obtained from Subgraph 1

Having obtained $\mathbf{V}_{\mathbf{s}_{k}}^{\left(i_{t}\right)}$ in (21), the a posteriori variance of the approximated noise $\xi_{k}$ is given by

$$
V_{\xi_{k}}^{\left(i_{t}\right)}=\phi_{k-1}^{T} \mathbf{V}_{\mathbf{s}_{k}}^{\left(i_{t}\right)}\left(\phi_{k-1}^{T}\right)^{H} .
$$

It is noted that the mean of the noise is zero and only the covariance has to be exchanged between Subgraph 1 and
Subgraph 2. According to (36) and the forward message $\vec{V}_{\xi_{k}}^{\left(i_{t}-1\right)}$, the backward message $\overleftarrow{V}_{\xi_{k}}^{\left(i_{t}\right)}$ from Subgraph 1 to Subgraph 2 is derived as

$$
\overleftarrow{V}_{\xi_{k}}^{\left(i_{t}\right)}=\frac{V_{\xi_{k}}^{\left(i_{t}\right)} \vec{V}_{\xi_{k}}^{\left(i_{t}-1\right)}}{\vec{V}_{\xi_{k}}^{\left(i_{t}-1\right)}-V_{\xi_{k}}^{\left(i_{t}\right)}}
$$

Based on (33) and (35), we can compute the a posteriori variance of the state vector $\boldsymbol{\xi}_{k}$ as

$$
\mathbf{V}_{\boldsymbol{\xi}_{k}}^{\left(i_{t}\right)}=\left(\left(\overrightarrow{\mathbf{V}}_{\boldsymbol{\xi}_{k}}^{\left(i_{t}\right)}\right)^{-1}+\overleftarrow{\mathbf{W}}_{\boldsymbol{\xi}_{k}}^{\left(i_{t}\right)}\right)^{-1}
$$

Then, we can derive the forward message of the $k$-th element of vector $\boldsymbol{\xi}_{k}$ from Subgraph 2 to Subgraph 1 as

$$
\vec{V}_{\xi_{k}}^{\left(i_{t}\right)}=\frac{\overleftarrow{V}_{\xi_{k}}^{\left(i_{t}\right)} \boldsymbol{\zeta}_{2} \mathbf{V}_{\boldsymbol{\xi}_{k}}^{\left(i_{t}\right)} \boldsymbol{\zeta}_{2}^{H}}{\overleftarrow{V}_{\xi_{k}}^{\left(i_{t}\right)}-\boldsymbol{\zeta}_{2} \mathbf{V}_{\boldsymbol{\xi}_{k}}^{\left(i_{t}\right)} \boldsymbol{\zeta}_{2}^{H}}
$$

The proposed hybrid GMP-EP equalization algorithm for SEFDM-IM systems with known CSI is summarized in Algorithm 1 , where $I_{\text {in }}$ and $I_{\text {out }}$ are the number of the inner and outer iterations, respectively.

Algorithm 1 Hybrid GMP-EP Equalization of SEFDM-IM Signaling with Known CSI

1: Initialization: The extrinsic LLR of the channel decoder is initialized as $L_{\mathrm{dec}}^{\mathrm{e},(0)}\left(c_{g, p}\right)=0, g=1, \cdots, G, p=$ $1, \cdots, P$. Then the $a$ priori mean and variance of transmitted symbols are $\vec{m}_{x_{k}}^{(0)}=0$ and $\vec{V}_{x_{k}}^{(0)}=+\infty, k=$ $0, \cdots, N_{s}-1$. The forward and backward messages are initialized as $\overrightarrow{\mathbf{m}}_{\mathbf{s}_{0}}^{\left(i_{t}\right)}=\mathbf{0}, \overrightarrow{\mathbf{V}}_{\mathbf{s}_{0}}^{\left(i_{t}\right)}=\mathbf{I}, \overrightarrow{\mathbf{V}}_{\boldsymbol{\xi}_{0}}^{\left(i_{t}\right)}=\mathbf{I}$, $\overleftarrow{\mathbf{W}}_{\mathbf{s}_{0}}^{\left(i_{t}\right)}=\mathbf{I}, \overleftarrow{\mathbf{W}}_{\mathbf{s}_{0}}^{\left(i_{t}\right)} \overleftarrow{\mathbf{m}}_{\mathbf{s}_{0}}^{\left(i_{t}\right)}=\mathbf{0}$, and $\overleftarrow{\mathbf{W}}_{\boldsymbol{\xi}_{0}}^{\left(i_{t}\right)}=\mathbf{I}$

2: for $i_{o}=1$ to $I_{\text {out }}$ do

3: $\quad$ for $i_{t}=1$ to $I_{\text {in }}$ do

4: $\quad$ Compute the backward messages of variable $\xi_{k}, k=$ $0, \cdots, N_{s}-1$ from Subgraph 1 to Subgraph 2 according to (36) and (37);

5: Compute the forward and backward mean vector as well as the covariance matrix on Subgraph 2 according to (32)-(33) and (34)-(35);

6: $\quad$ Compute the forward covariance matrix of variable $\xi_{k}, k=0, \cdots, N_{s}-1$, from Subgraph 2 to Subgraph 1 according to (38) and (39);

7: $\quad$ Compute the outgoing messages $\overleftarrow{m}_{x_{k}}^{\left(i_{t}\right)}$ and $\overleftarrow{V}_{x_{k}}^{\left(i_{t}\right)}$ according to (29)-(31);

8: $\quad$ Compute the approximated Gaussian incoming messages based on the a priori possibilities and the outgoing Gaussian messages according to (25)-(28).

\section{9: $\quad$ end for}

10: Compute the extrinsic LLRs of the equalizer based on the outgoing messages and feed them to the channel decoder;

11: Perform BCJR channel decoding and compute the discrete a priori possibilities of the transmitted symbols based on the extrinsic LLRs of the channel decoder using (22)-(23).

2: end for 


\section{GMP-EP-VMP JOINT CHANNEL ESTIMATION AND EQUALIZATION}

In contrast to OFDM-based systems, a typical linear MMSE channel estimator designed for SEFDM-IM signaling suffers from an excessive computational complexity due to the matrix inversion of the inherent interference matrix. In this section, we develop a low-complexity joint FD channel estimation and equalization method for SEFDM-IM systems. By appropriately grouping the elements of the channel's frequency responses, we reformulate the channel estimator via a linear state-space model and connect it with the equalizer described in Section III via an inner product node. To tackle error propagation during channel estimation on the deactivated subcarriers, we exploit the channel characteristics for constructing a multi-layer factor graph. Moreover, we derive the CRLB for the proposed channel estimator in closed-form.

\section{A. Factor Graph Model for SEFDM-IM Systems Having Un- known CSI}

Consider a $L$-tap frequency-selective fading channel, where the number of subcarriers is $N_{s}=Q L$, where $Q$ is a positive integer. By grouping the elements of the FD channel response, we obtain $Q$ vectors $\breve{\mathbf{h}}_{q}=\left[h_{q}, h_{Q+q}, \cdots, h_{(L-1) Q+q}\right]^{T}, q=$ $0, \cdots, Q-1$. Each vector $\mathbf{h}_{q}$ consists of $L$ FD channel response samples and can be obtained via non-orthogonal transformation of the CIR as $\breve{\mathbf{h}}_{q}=\boldsymbol{\Gamma}_{L}\left(\boldsymbol{\Lambda}^{H}\right)^{q} \overline{\mathbf{h}}$, where $\boldsymbol{\Gamma}_{L}$ is a non-orthogonal transformation matrix with $\Gamma_{m, n}=e^{-\frac{j 2 \pi \alpha m n}{L}}$ and $\boldsymbol{\Lambda}=\mathcal{D}\left(\left[1, e^{\frac{j 2 \pi \alpha}{N_{s}}}, \cdots, e^{\frac{j 2 \pi \alpha(L-1)}{N_{s}}}\right]^{T}\right)$. Thus, the state transition equation is given by

$$
\breve{\mathbf{h}}_{q-1}=\boldsymbol{\Gamma}_{L} \boldsymbol{\Lambda} \boldsymbol{\Gamma}_{L}^{-1} \breve{\mathbf{h}}_{q} .
$$

Assuming that the CSI remains unchanged during $\tilde{L}$ SEFDM-IM symbols, the corresponding $q$-th transmitted symbol vector of the $\tilde{l}$-th SEFDM-IM symbol is denoted as $\breve{\mathbf{s}}_{q}^{\tilde{l}}=\left[s_{q}^{\tilde{l}}, s_{Q+q}^{\tilde{l}}, \cdots, s_{(L-1) Q+q}^{\tilde{l}}\right]^{T}$. The transmitted SEFDMIM symbols and the frequency-domain channel response satisfy $\breve{\mathbf{s}}_{q}^{\tilde{l}}=\breve{\mathbf{h}}_{q}^{\tilde{l}} \odot \breve{\mathbf{x}}_{q}^{\tilde{l}}$. Each element of $\breve{\mathbf{s}}_{q}^{\tilde{l}}$ is represented as $s_{l}^{\tilde{l}}=\boldsymbol{v}_{l}^{T} \breve{\mathbf{s}}_{q}^{\tilde{l}}$, where $\boldsymbol{v}_{l}$ is an indicator vector with the $l$-th element being one.

With the assumption of an unknown CSI, channel factor node in Fig. 2 is replaced by the FD channel estimator feeding the equalizer. For quasi-static channels, different layers are connected via channel variable nodes on the same subcarrier positions of different symbols. Based on the above model, the $\tilde{L}$-sublayer factor graph representation of our joint channel estimation and SEFDM-IM equalization relying on unknown CSI is depicted in Fig. 3. The multiplier node $\nabla$ represents the inner product constraint $\delta\left(\breve{\mathbf{s}}_{q}^{\tilde{l}}-\breve{\mathbf{h}}_{q}^{l} \odot \breve{\mathbf{x}}_{q}^{\tilde{l}}\right)$.

\section{B. VMP-based Channel Estimation}

The a priori information gleaned from channel decoder is computed as (25)-(28). We express the approximated Gaussian

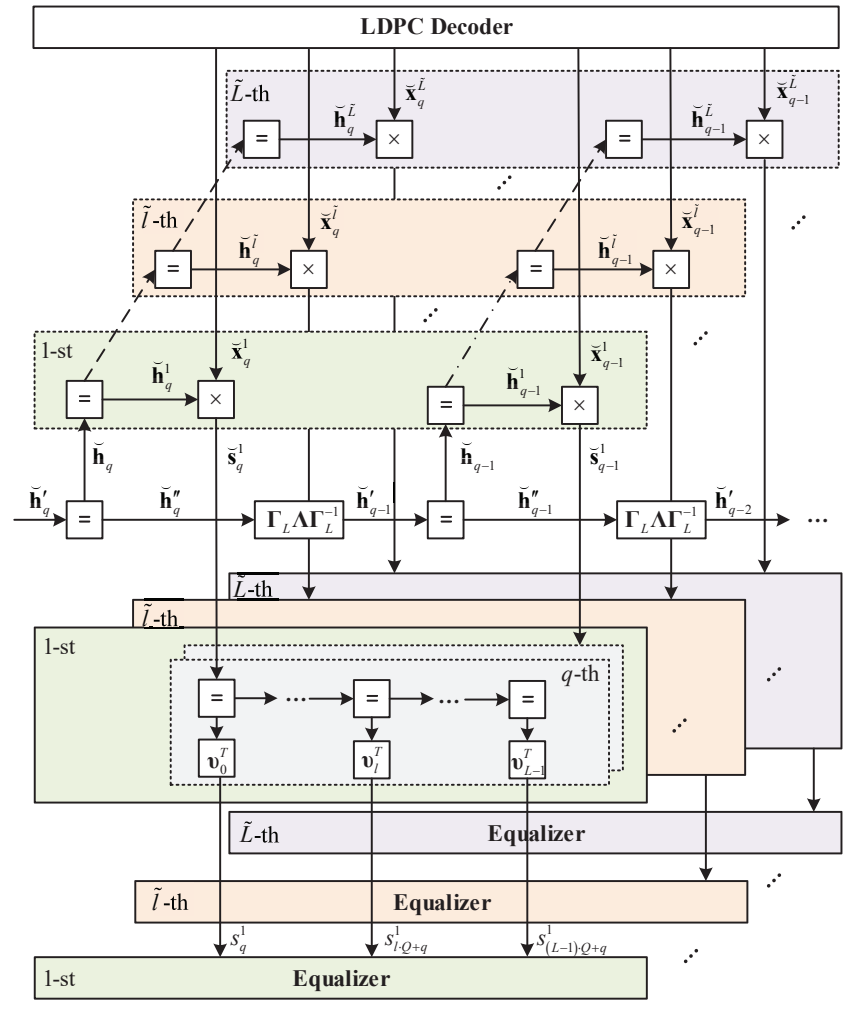

Fig. 3. Factor graph for joint channel estimation and equalization of SEFDMIM signaling with unknown CSI. The equalizer contains Subgraph 1 and Subgraph 2 in Fig. 2.

messages passed from the channel estimator to the equalizer as

$$
\begin{aligned}
& \vec{m}_{s_{k}^{i}}^{\left(i_{t}\right)}=\boldsymbol{v}_{l}^{T}\left(\overrightarrow{\mathbf{m}}_{\breve{\mathbf{x}}_{q}^{\tilde{l}}}^{\left(i_{t}\right)} \odot \overrightarrow{\mathbf{m}}_{\breve{\mathbf{h}}_{q}^{\tilde{l}}}^{\left(i_{t}\right)}\right), \\
& \vec{V}_{s_{k}^{\tilde{l}}}^{\left(i_{t}\right)}=\boldsymbol{v}_{l}^{T}\left[\mathcal{D}\left(\overrightarrow{\mathbf{m}}_{\widetilde{\mathbf{x}}_{q}^{\tilde{l}}}^{\left(i_{t}\right)} \odot \overrightarrow{\mathbf{m}}_{\widetilde{\mathbf{x}}_{q}^{\tilde{I}}}^{\left(i_{t}\right)}\right)+\overrightarrow{\mathbf{V}}_{\widetilde{\mathbf{x}}_{q}^{\tilde{l}}}^{\left(i_{t}\right)}\right] \\
& \times\left[\mathcal{D}\left(\overrightarrow{\mathbf{m}}_{\breve{\mathbf{h}}_{q}^{\tilde{\tau}}}^{\left(i_{t}\right)} \odot \overrightarrow{\mathbf{m}}_{\breve{\mathbf{h}}_{q}^{\tilde{l}}}^{\left(i_{t}\right)}\right)+\overrightarrow{\mathbf{V}}_{\breve{\mathbf{h}}_{q}^{\tilde{\tau}}}^{\left(i_{t}\right)}\right]\left(\boldsymbol{v}_{l}^{T}\right)^{H}-\left(\vec{m}_{s_{k}^{\tau}}^{\left(i_{t}\right)}\right)^{2},
\end{aligned}
$$

where the forward messages $\overrightarrow{\mathbf{m}}_{\mathbf{h}_{q}^{i}}^{\left(i_{t}\right)}$ and $\overrightarrow{\mathbf{V}}_{\mathbf{h}_{q}^{i}}^{\left(i_{t}\right)}$ depend on the channel estimates at the $\left(i_{t}-1\right)$-st iteration. The detailed derivations of (41) and (42) are given in Appendix A.

The backward messages of variables $\breve{\mathbf{s}}_{q}^{l}$ consist of the corresponding outgoing messages of the equalizer. For the inner product node, message updating using classical BP is intractable. To derive approximated Gaussian expressions, the backward message of $\tilde{\mathbf{h}}_{q}^{\tilde{l}}$ from the inner product node is updated according to the VMP rules of [42], [43] as

$$
\overleftarrow{\mu}\left(\breve{\mathbf{h}}_{q}^{\tilde{l}}\right) \propto \exp \left(\int \ln \delta\left(\breve{\mathbf{s}}_{q}^{\tilde{l}}-\mathcal{D}\left(\breve{\mathbf{x}}_{q}^{\tilde{l}}\right) \breve{\mathbf{h}}_{q}^{\tilde{l}}\right) b\left(\breve{\mathbf{x}}_{q}^{\tilde{l}}\right) b\left(\breve{\mathbf{s}}_{q}^{\tilde{l}}\right) d \breve{\mathbf{x}}_{q}^{\tilde{l}} d \breve{\mathbf{s}}_{q}^{\tilde{l}}\right)
$$

where $b\left(\breve{\mathbf{x}}_{q}^{\tilde{l}}\right)$ and $b\left(\breve{\mathbf{s}}_{q}^{\tilde{l}}\right)$ are the beliefs of the variables $\breve{\mathbf{x}}_{q}^{\tilde{l}}$ and $\breve{\mathbf{s}}_{q}^{\tilde{l}}$, respectively.

It is noted that the logarithm of the delta function involved in the integration of (43) is pathological [40]. To this end, the multiplier node $\nabla$ is grouped with the edge $\breve{\mathbf{s}}_{q}^{\tilde{l}}$ as an equivalent soft node $f_{q}^{\tilde{l}}\left(\breve{\mathbf{x}}_{q}^{\tilde{l}}, \breve{\mathbf{h}}_{q}^{\tilde{l}}\right) \propto \exp \left[-\left(\mathcal{D}\left(\breve{\mathbf{x}}_{q}^{\tilde{l}}\right) \breve{\mathbf{h}}_{q}^{l}-\right.\right.$ $\left.\left.\overleftarrow{\mathbf{m}}_{\breve{\mathbf{s}}_{q}^{\tilde{l}}}^{\left(i_{t}\right)}\right)^{H}\left(\overleftarrow{\mathbf{V}}_{\breve{\mathbf{s}}_{q}^{\tilde{l}}}^{\left(i_{t}\right)}\right)^{-1}\left(\mathcal{D}\left(\breve{\mathbf{x}}_{q}^{\tilde{l}}\right) \breve{\mathbf{h}}_{q}^{\tilde{l}}-\overleftarrow{\mathbf{m}}_{\breve{\mathbf{s}}_{q}^{\tilde{l}}}^{\left(i_{t}\right)}\right)\right]$. Then, the backward 
messages of the variables $\breve{\mathbf{h}}_{q}^{\tilde{l}}$ are derived in Appendix B. The backward mean vector and covariance matrix of variables $\breve{\mathbf{h}}_{q}^{\tilde{l}}$ are given by

$\overleftarrow{\mathbf{m}}_{\tilde{\mathbf{h}}_{q}^{\tau}}^{\left(i_{t}\right)}=\mathcal{D}\left(\left(\mathbf{m}_{\widetilde{\mathbf{x}}_{q}^{\tilde{l}}}^{\left(i_{t}\right)}\right)^{H} \odot \overleftarrow{\mathbf{m}}_{\widetilde{\mathbf{s}}_{q}^{\tilde{l}}}^{\left(i_{t}\right)}\right)\left[\mathbf{V}_{\widetilde{\mathbf{x}}_{q}^{\tilde{l}}}^{\left(i_{t}\right)}+\mathcal{D}\left(\left(\mathbf{m}_{\widetilde{\mathbf{x}}_{q}^{\tilde{l}}}^{\left(i_{t}\right)}\right)^{H} \odot \mathbf{m}_{\widetilde{\mathbf{x}}_{q}^{\tilde{l}}}^{\left(i_{t}\right)}\right)\right]^{-1}$

$\overleftarrow{\mathbf{V}}_{\breve{\mathbf{h}}_{q}^{\tilde{l}}}^{\left(i_{t}\right)}=\overleftarrow{\mathbf{V}}_{\widetilde{\mathbf{s}}_{q}^{\tilde{l}}}^{\left(i_{t}\right)}\left[\mathbf{V}_{\widetilde{\mathbf{x}}_{q}^{\tilde{l}}}^{\left(i_{t}\right)}+\mathcal{D}\left(\left(\mathbf{m}_{\widetilde{\mathbf{x}}_{q}^{\tilde{l}}}^{\left(i_{t}\right)}\right)^{H} \odot \mathbf{m}_{\widetilde{\mathbf{x}}_{q}^{\tilde{l}}}^{\left(i_{t}\right)}\right)\right]^{-1}$

where both $\mathbf{V}_{\breve{\mathbf{x}}_{q}^{\tilde{\tau}}}^{\left(i_{t}\right)}$ and $\overleftarrow{\mathbf{V}}_{\widetilde{\mathbf{s}}_{q}^{\tilde{\tau}}}^{\left(i_{t}\right)}$ are diagonal matrices. The a posteriori mean vector and covariance matrix of $\breve{\mathbf{x}}_{q}^{\tilde{l}}$ are formulated as

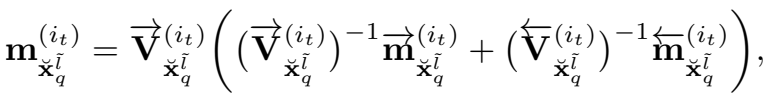

$$
\begin{aligned}
& \mathbf{V}_{\tilde{\mathbf{x}}_{q}^{\tilde{l}}}^{\left(i_{t}\right)}=\left(\left(\overrightarrow{\mathbf{V}}_{\widetilde{\mathbf{x}}_{q}^{\tilde{l}}\left(i_{t}\right)}^{\left(i^{\prime}\right.}\right)^{-1}+\left(\overleftarrow{\mathbf{V}}_{\tilde{\mathbf{x}}_{q}^{\tilde{I}}}^{\left(i_{t}\right)}\right)^{-1}\right)^{-1}
\end{aligned}
$$

Since the inner product constraint can also be represented as $\delta\left(\breve{\mathbf{s}}_{q}^{\tilde{l}}-\mathcal{D}\left(\breve{\mathbf{h}}_{q}^{\tilde{l}}\right) \breve{\mathbf{x}}_{q}^{\tilde{l}}\right)$, similar to the derivations of (44) and (45), the backward mean vector and covariance matrix of variables $\breve{\mathbf{x}}_{q}^{\tilde{l}}$ are derived as

$$
\begin{aligned}
& \overleftarrow{\mathbf{m}}_{\widetilde{\mathbf{x}}_{q}^{\tilde{\nu}}}^{\left(i_{t}\right)}=\mathcal{D}\left(\left(\mathbf{m}_{\breve{\mathbf{h}}_{q}^{\tilde{\tau}}}^{\left(i_{t}-1\right)}\right)^{H} \odot \overleftarrow{\mathbf{m}}_{\widetilde{\mathbf{s}}_{q}^{\tilde{l}}}^{\left(i_{t}\right)}\right) \\
& \times\left[\mathbf{V}_{\breve{\mathbf{h}}_{q}^{\tau}}^{\left(i_{t}-1\right)}+\mathcal{D}\left(\left(\mathbf{m}_{\breve{\mathbf{h}}_{q}^{\tau}}^{\left(i_{t}-1\right)}\right)^{H} \odot \mathbf{m}_{\breve{\mathbf{h}}_{q}^{\tau}}^{\left(i_{t}-1\right)}\right)\right]^{-1} \\
& \overleftarrow{\mathbf{V}}_{\breve{\mathbf{x}}_{q}^{\tilde{\tau}}}^{\left(i_{t}\right)}=\overleftarrow{\mathbf{V}}_{\breve{\mathbf{s}}_{q}^{\tilde{l}}}^{\left(i_{t}\right)}\left[\mathbf{V}_{\widetilde{\mathbf{h}}_{q}^{\tilde{\tau}}}^{\left(i_{t}-1\right)}+\mathcal{D}\left(\left(\mathbf{m}_{\breve{\mathbf{h}}_{q}^{\tilde{\tau}}}^{\left(i_{t}-1\right)}\right)^{H} \odot \mathbf{m}_{\widetilde{\mathbf{h}}_{q}^{\tilde{\tau}}}^{\left(i_{t}-1\right)}\right)\right]^{-1}
\end{aligned}
$$

where the a posteriori mean vector $\mathbf{m}_{\breve{\mathbf{h}}_{q}^{\tilde{\tau}}}^{\left(i_{t}-1\right)}$ and the covariance matrix $\mathbf{V}_{\breve{\mathbf{h}}_{q}^{\tilde{\tau}}}^{\left(i_{t}-1\right)}$ of $\breve{\mathbf{h}}_{q}^{\tilde{l}}$ can be obtained similarly to that in (46)(47). Therefore, the outgoing messages of $x_{k}^{\tilde{l}}$ are given by

$$
\begin{aligned}
& \overleftarrow{m}_{x_{k}^{\tau}}^{\left(i_{t}\right)}=\overleftarrow{m}_{x_{\left(l_{1}-1\right) Q+q}^{\tilde{l}}}^{\left(i_{t}\right)}=\boldsymbol{v}_{\boldsymbol{l}_{\mathbf{1}}}^{T} \overleftarrow{\mathbf{m}}_{\widetilde{\mathbf{x}}_{q}^{\tilde{l}}}^{\left(i_{t}\right)} \\
& \overleftarrow{V}_{x_{k}^{\tilde{I}}}^{\left(i_{t}\right)}=\overleftarrow{V}_{x_{\left(l_{1}-1\right) Q+q}^{\tilde{\tau}}}^{\left(i_{i^{\prime}}\right)}=\boldsymbol{v}_{\boldsymbol{l}_{\mathbf{1}}}^{T} \overleftarrow{\mathbf{V}}_{\overleftarrow{\mathbf{x}}_{q}^{\tilde{\tau}}}^{\left(i_{t}\right)} \boldsymbol{v}_{\boldsymbol{l}_{\mathbf{1}}}
\end{aligned}
$$

where $x_{k}^{\tilde{l}}$ is the $l_{1}$ element of $\breve{\mathbf{x}}_{q}^{\tilde{l}}$ and the subscript $k$ satisfies $k=\left(l_{1}-1\right) Q+q$.

Based on the backward messages of the variables $\breve{\mathbf{h}}_{q}^{\tilde{l}}$ in the $\tilde{l}$-th sublayer, the GMP rules concerning equality nodes are employed for determining the messages exchanged between different sublayers. The backward weight matrices and the transformed mean vectors related to the same subcarriers are added together to produce the backward messages of $\breve{\mathbf{h}}_{q}$, yielding,

$$
\begin{aligned}
& \overleftarrow{\mathbf{V}}_{\breve{\mathbf{h}}_{q}}^{\left(i_{t}\right)}=\left(\sum_{\breve{l}=1}^{\breve{L}} \overleftarrow{\mathbf{W}}_{\breve{\mathbf{h}}_{q}^{\bar{l}}}^{\left(i_{t}\right)}\right)^{-1}, \\
& \overleftarrow{\mathbf{m}}_{\breve{\mathbf{h}}_{q}}^{\left(i_{t}\right)}=\overleftarrow{\mathbf{V}}_{\breve{\mathbf{h}}_{q}}^{\left(i_{t}\right)} \sum_{\breve{l}=1}^{\breve{L}} \overleftarrow{\mathbf{W}}_{\breve{\mathbf{h}}_{q}^{l}}^{\left(i_{t}\right)} \overleftarrow{\mathbf{m}}_{\breve{\mathbf{h}}_{q}^{\tau}}^{\left(i_{t}\right)},
\end{aligned}
$$

where $\overleftarrow{\mathbf{W}}_{\overleftarrow{\mathbf{h}}_{q}^{\imath}}^{\left(i_{t}\right)}=\left(\overleftarrow{\mathbf{V}}_{\breve{\mathbf{h}}_{q}^{i}}^{\left(i_{t}\right)}\right)^{-1}$. Then the forward messages of the state $\mathbf{h}_{q}^{\prime \prime}$ are also computed as

$$
\begin{aligned}
& \overrightarrow{\mathbf{m}}_{\breve{\mathbf{h}}_{q}^{\prime \prime}}^{\left(i_{t}\right)}=\overrightarrow{\mathbf{V}}_{\breve{\mathbf{h}}_{q}^{\prime \prime}}^{\left(i_{t}\right)}\left(\left(\overrightarrow{\mathbf{V}}_{\breve{\mathbf{h}}_{q}^{\prime}}^{\left(i_{t}\right)}\right)^{-1} \overrightarrow{\mathbf{m}}_{\breve{\mathbf{h}}_{q}^{\prime}}^{\left(i_{t}\right)}+\left(\overleftarrow{\mathbf{V}}_{\breve{\mathbf{h}}_{q}}^{\left(i_{t}\right)}\right)^{-1} \overleftarrow{\mathbf{m}}_{\breve{\mathbf{h}}_{q}}^{\left(i_{t}\right)}\right), \\
& \overrightarrow{\mathbf{V}}_{\breve{\mathbf{h}}_{q}^{\prime \prime}}^{\left(i_{t}\right)}=\left(\left(\overrightarrow{\mathbf{V}}_{\breve{\mathbf{h}}_{q}^{\prime}}^{\left(i_{t}\right)}\right)^{-1}+\left(\overleftarrow{\mathbf{V}}_{\breve{\mathbf{h}}_{q}}^{\left(i_{t}\right)}\right)^{-1}\right)^{-1} .
\end{aligned}
$$

The initial messages for the edge representing $\breve{\mathbf{h}}_{Q-1}^{\prime}$ can be obtained by using pilots based on the TD channel estimation method [20]. Hence, the forward messages of the state $\mathbf{h}_{q-1}^{\prime}$ are expressed as

$$
\begin{aligned}
& \overrightarrow{\mathbf{m}}_{\breve{h}_{q-1}^{\prime}}^{\left(i_{t}\right)}=\boldsymbol{\Gamma}_{L} \boldsymbol{\Lambda} \boldsymbol{\Gamma}_{L}^{-1} \overrightarrow{\mathbf{m}}_{\breve{\mathbf{h}}_{q}^{\prime \prime}}^{\left(i_{t}\right)}, \\
& \overrightarrow{\mathbf{V}}_{\breve{\mathbf{h}}_{q-1}^{\prime}\left(i_{t}\right)}^{\prime \prime}=\boldsymbol{\Gamma}_{L} \boldsymbol{\Lambda} \boldsymbol{\Gamma}_{L}^{-1} \overrightarrow{\mathbf{V}}_{\breve{\mathbf{h}}_{q}^{\prime \prime}}^{\left(i_{t}\right)} \boldsymbol{\Gamma}_{L}^{-H} \boldsymbol{\Lambda}^{H} \boldsymbol{\Gamma}_{L}^{H} .
\end{aligned}
$$

Obviously, the variance matrix $\overrightarrow{\mathbf{V}}_{\breve{\mathbf{h}}_{q-1}^{\prime}}^{\left(i_{t}\right)}$ is no longer diagonal in (57), which substantially increases the complexity of the proposed algorithm. To reduce the computation complexity, we approximate the variance matrix as

$$
\overrightarrow{\mathbf{V}}_{\overrightarrow{\mathbf{h}}_{q}^{\prime}}^{\left(i_{t}\right)}=\frac{1}{L} \operatorname{tr}\left(\overrightarrow{\mathbf{V}}_{\overrightarrow{\mathbf{h}}_{q}^{\prime \prime}}^{\left(i_{t}\right)}\right) \mathbf{I}_{L}
$$

Based on (58), we can express the forward messages of $\breve{\mathbf{h}}_{0}^{\prime}$ at the rightmost side of the factor graph. Upon combining with the backward messages in (44) and (45), we have

$$
\begin{aligned}
& \mathbf{m}_{\breve{\mathbf{h}}_{0}}^{\left(i_{t}\right)}=\mathbf{V}_{\breve{\mathbf{h}}_{0}}^{\left(i_{t}\right)}\left(\left(\overrightarrow{\mathbf{V}}_{\breve{\mathbf{h}}_{0}}^{\left(i_{t}\right)}\right)^{-1} \overrightarrow{\mathbf{m}}_{\breve{\mathbf{h}}_{0}}^{\left(i_{t}\right)}+\left(\overleftarrow{\mathbf{V}}_{\breve{\mathbf{h}}_{0}}^{\left(i_{t}\right)}\right)^{-1} \overleftarrow{\mathbf{m}}_{\breve{\mathbf{h}}_{0}}^{\left(i_{t}\right)}\right), \\
& \mathbf{V}_{\breve{\mathbf{h}}_{0}}^{\left(i_{t}\right)}=\left(\left(\overrightarrow{\mathbf{V}}_{\breve{\mathbf{h}}_{0}}^{\left(i_{t}\right)}\right)^{-1}+\left(\overleftarrow{\mathbf{V}}_{\breve{\mathbf{h}}_{0}}^{\left(i_{t}\right)}\right)^{-1}\right)^{-1} .
\end{aligned}
$$

Since the a posteriori messages are identical on the equation nodes, the channel estimates at the $i_{t}$-th inner iteration can be updated as

$$
\begin{aligned}
& \mathbf{m}_{\breve{\mathbf{h}}_{q}^{i}}^{\left(i_{t}\right)}=\mathbf{m}_{\breve{\mathbf{h}}_{q}}^{\left(i_{t}\right)}=\boldsymbol{\Gamma}_{L} \boldsymbol{\Lambda}^{H} \boldsymbol{\Gamma}_{L}^{-1} \mathbf{m}_{\breve{\mathbf{h}}_{q-1}}^{\left(i_{t}\right)}, \\
& \mathbf{V}_{\breve{\mathbf{h}}_{q}^{i}}^{\left(i_{t}\right)}=\mathbf{V}_{\breve{\mathbf{h}}_{q}}^{\left(i_{t}\right)}=\boldsymbol{\Gamma}_{L} \boldsymbol{\Lambda}^{H} \boldsymbol{\Gamma}_{L}^{-1} \mathbf{V}_{\breve{\mathbf{h}}_{q-1}}^{\left(i_{t}\right)} \boldsymbol{\Gamma}_{L}^{-H} \boldsymbol{\Lambda} \boldsymbol{\Gamma}_{L}^{H} .
\end{aligned}
$$

Note that the product of $\boldsymbol{\Gamma}_{L}$ (or $\boldsymbol{\Gamma}_{L}^{H}$ ) with a vector in (56) and (62) can be efficiently implemented using a single $(L / \alpha)$ point IDFT, when $L / \alpha$ is an integer or using $c$ parallel $L$-point IDFTs with $c L$ complex multiplications when $\alpha$ is a rational number [32].

In order to illustrate the reliability of the proposed GMPEP-VMP method, we derive the Cramer-Rao lower bound (CRLB) for the proposed channel estimator in Appendix C. Furthermore, the normalized mean square error (NMSE) of the proposed channel estimator satisfies

$$
\mathrm{NMSE}=\mathbb{E}\left\{\frac{\|\overline{\mathbf{h}}-\hat{\overline{\mathbf{h}}}\|^{2}}{\|\overline{\mathbf{h}}\|^{2}}\right\} \geq \mathrm{CRLB}=\operatorname{tr}\left[\mathbf{I}^{-1}(\overline{\mathbf{h}})\right],
$$

where $\mathbf{I}(\overline{\mathbf{h}})$ is the Fisher information matrix in (71) and $\|\overline{\mathbf{h}}\|^{2}=1$.

The proposed hybrid GMP-EP-VMP joint channel estimation and equalization algorithm for SEFDM-IM systems communicating over frequency-selective fading channels is summarized in Algorithm 2, where $I_{\mathrm{in}}$ and $I_{\text {out }}$ are the number of the inner and outer iterations, respectively. 


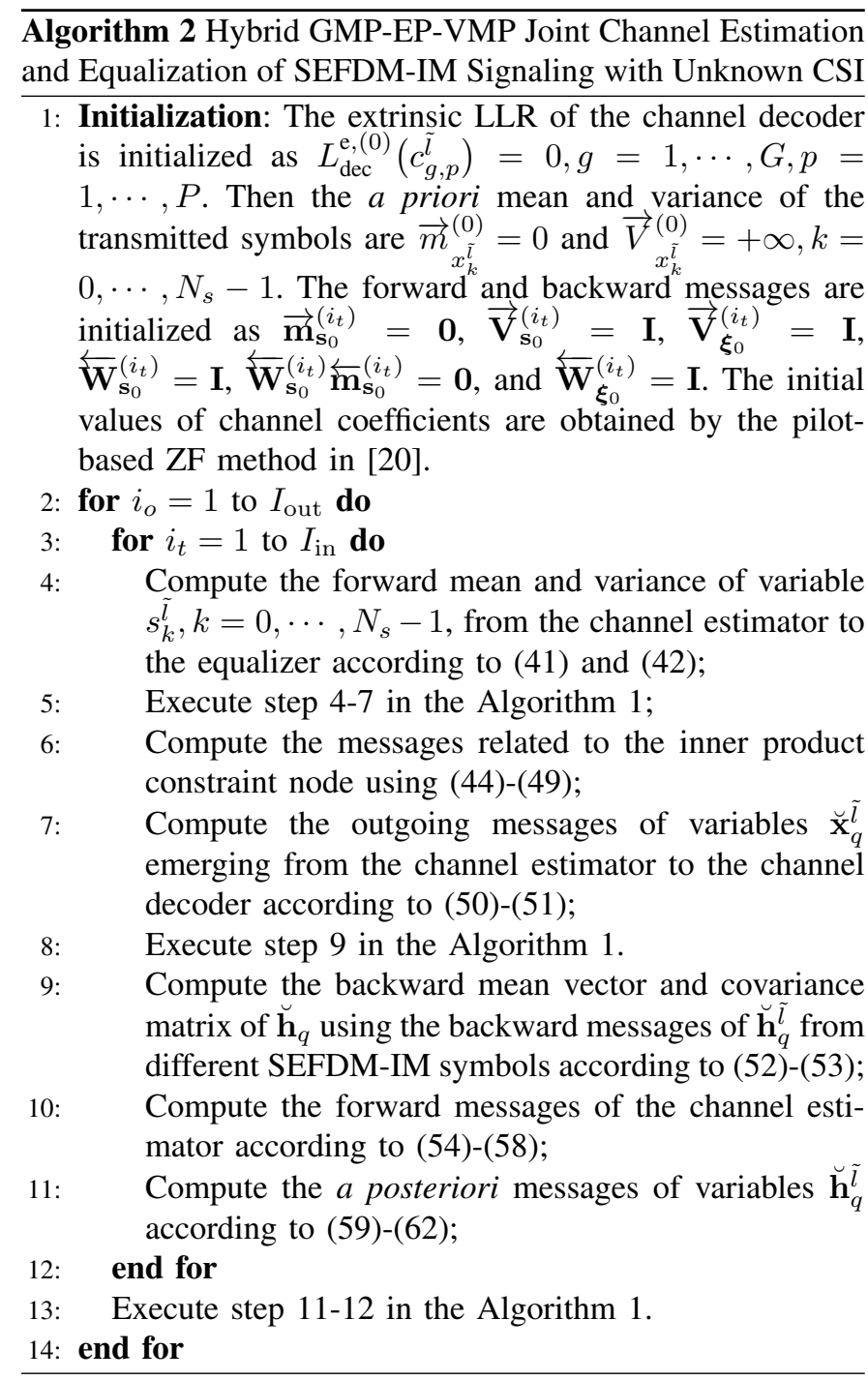

\section{Complexity Analysis}

The complexity comparison of the proposed GMP-EP and GMP-EP-VMP algorithms to that of the existing methods is summarized in Table I and Table II, respectively. Since the standard soft information calculation and BCJR decoding are performed in all methods in the following, we only focus our attention on the complexity of the equalizer and channel estimator. The optimal MAP equalizer suffers from an exponentially increased complexity order of $\mathcal{O}\left(2^{P G}\right)$, where $P$ is the number of coded bits per subblock and $G$ is the total number of subblocks. The complexity of the BML method approximately adopted from [28] exploits an ML detector having a reduced search space and a complexity order of $\mathcal{O}\left(2^{P\left\lceil\frac{L_{m}}{N}\right\rceil}\right)$, where $L_{m}$ is the truncated length of the interferences for the exhaustive search and $N$ is the number of SEFDM-IM subcarriers per subblock. The improved MMSELLR method suitably adopted from [30] consists of the MMSE equalizer and the LLR-based detector having an extended joint search space, a complexity order of $\mathcal{O}\left(N_{s}^{3}\right)$ and $\mathcal{O}\left(G M^{2}\right)$, respectively, where $N_{s}$ is the total number of SEFDM-IM subcarriers and $M$ represents the $M$-ary constellation mapping. When relying on IFFT/FFT-based modulation/demodulation,
TABLE I

COMPLEXITY ANALYSIS OF EQUALIZER

\begin{tabular}{|l|r|}
\hline Algorithm & Complexity \\
\hline ML & $\mathcal{O}\left(2^{P G}\right)$ \\
\hline BML & $\mathcal{O}\left(2^{\left.P \Gamma \frac{L_{m}}{N}\right\rceil}\right)$ \\
\hline MMSE-SBS & $\mathcal{O}\left(N_{s}^{3}\right)$ \\
\hline MMSE-LLR & $\mathcal{O}\left(N_{s}^{3}\right)+\mathcal{O}\left(G M^{2}\right)$ \\
\hline SIC-SBS & $\mathcal{O}\left(3 c N_{s} \log _{2} N_{s}\right)$ \\
\hline GMP-EP & $\mathcal{O}\left(N_{s} L_{s}^{2}\right), L_{s} \ll N_{s}$ \\
\hline
\end{tabular}

TABLE II

COMPLEXITY ANALYSIS OF EQUALIZER AND CHANNEL ESTIMATOR

\begin{tabular}{|l|r|r|}
\hline \multirow{2}{*}{ Algorithm } & \multicolumn{2}{|c|}{ Complexity } \\
\cline { 2 - 3 } & $\mathcal{O}\left(3 c N_{s} \log _{2} N_{s}\right)$ & Channel Estimator \\
\hline T-FCE/PCE-SIC & $\mathcal{O}\left(3 c N_{s} \log _{2} N_{s}\right)$ & $\mathcal{O}\left(N_{s}^{3}\right)$ \\
\hline F-PCE-SIC & $\mathcal{O}\left(N_{s}\right)$ \\
\hline GMP-EP-VMP & $\mathcal{O}\left(N_{s} L_{s}^{2}\right), L_{s} \ll N_{s}$ & $\mathcal{O}\left(c N_{s} \log _{2} L\right)+\mathcal{O}\left(N_{s} L\right)$ \\
\hline
\end{tabular}

the complexity of the SIC-SBS equalizer extended from [18] and [44] grows logarithmically with the length of FFT. For the proposed GMP-EP equalizer, the complexity is dominated by the matrix inversion operation in (29) and (21), having a complexity order of $\mathcal{O}\left(L_{s}^{3}\right)$, where $L_{s}$ is the total truncated length of interferences considered for ICI mitigation. Since it is computed $N_{s} / L_{s}$ times per iteration, the total complexity of the proposed GMP-EP equalizer is on the order of $\mathcal{O}\left(N_{s} L_{s}^{2}\right)$ per iteration. Compared to the existing methods, the proposed GMP-EP equalizer is preferable for SEFDM-IM systems having a large number of subcarriers and/or high-order modulation.

For the channel estimation, the classical time-domain pilotbased full channel estimator (FCE) [20] and partial channel estimator (PCE) [21] channel estimators are derived based on the ZF criterion, having a complexity order of $\mathcal{O}\left(N_{s}^{3}\right)$. In [22], the FD PCE using SEFDM pilots is derived, which has a linearly increasing complexity vs the number of subcarriers, plus the additional interpolation complexity. The above channel estimator can be combined with an SIC equalizer for constructing an SEFDM-IM receiver. The variance matrices of the proposed hybrid GMP-EP-VMP algorithm are approximated by diagonal matrices using the trace operation in (57), which results in trivial complexity for the matrix inversion. The inversion operation of the non-diagonal matrix $\boldsymbol{\Gamma}_{L}$ only has to be calculated once, which can be performed off-line. As a result, the complexity of the GMP-EP-VMP method is dominated by the matrix product operation related to $\boldsymbol{\Gamma}_{L}$, which can be implemented by the IFFT/FFT operation. The complexity of the other matrix product operations depends on the number of subcarriers and on the length of the channel memory per iteration. Therefore, the total complexity of the proposed channel estimator is $\mathcal{O}\left(c N_{s} \log _{2} L\right)+\mathcal{O}\left(N_{s} L\right)$ for $\alpha=\frac{b}{c}, b, c \in \mathbb{N}$ per iteration, where $\alpha$ is the subcarrier packing factor, $L$ is the length of channel memory and $N_{s}=Q L$.

\section{Simulation Results and Discussions}

We now evaluate both the BER and NMSE performance of the proposed methods by Monte Carlo simulations. In all simulations, we employ a LDPC code having code rate 
TABLE III

THE NORMALIZED PDP OF THE RICIAN FADING CHANNEL HAVING $K_{h}=10 d B$

\begin{tabular}{|l|c|c|c|c|c|c|c|c|}
\hline Tap & 1 & 2 & 3 & 4 & 5 & 6 & 7 & 8 \\
\hline Normalized Delay & 0.0 & 0.1 & 0.2 & 0.3 & 0.4 & 0.5 & 0.6 & 0.7 \\
\hline Normalized Power & 0.9000 & 0.0189 & 0.0171 & 0.0155 & 0.0140 & 0.0127 & 0.0115 & 0.0104 \\
\hline
\end{tabular}

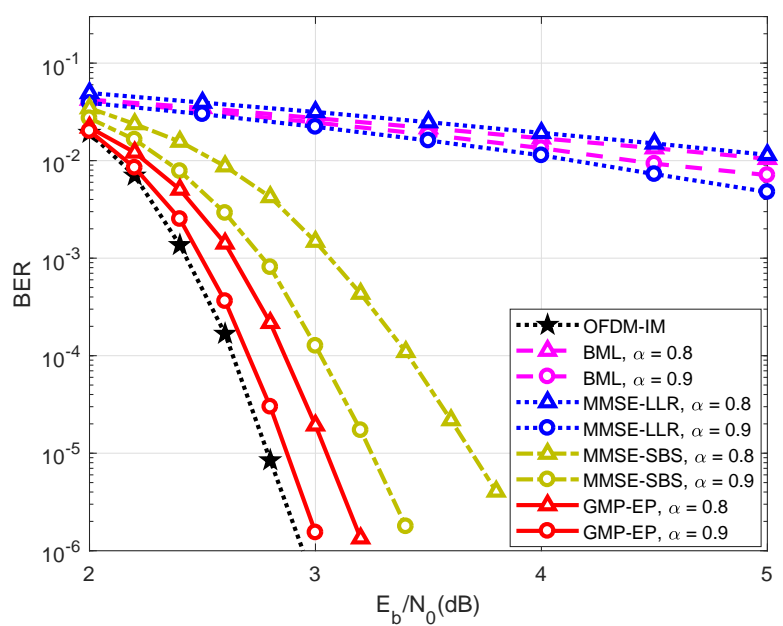

Fig. 4. BER performance of different equalizers for SEFDM-IM systems with known CSI, $(N, K)=(4,1)$.

of $r=3312 / 4032$ and code length of 4032 bits, unless otherwise specified. In [24], it is demonstrated that subcarrierindex modulation is beneficial for the scenario of a relatively low transmission rate below 2 bits $/ \mathrm{s} / \mathrm{Hz}$. Hence, we only employ QPSK modulation using Gray mapping in this paper. Each frame consists of $\tilde{L}=32$ SEFDM-IM symbols and pilots are only transmitted on the first symbol. For notational simplicity, we refer to the SEFDM-IM scheme with $K$ out of $N$ subcarriers being active per subblock as the $(N, K)$ scheme. The order of CAR model is set to $\bar{P}=1$. The truncated length of interferences is $L_{t}=5$, unless otherwise specified. A Rician fading channel having $L=8$ paths and a Rician factor of $K_{h}=10 \mathrm{~dB}$ is studied [45]. The coefficient $\bar{h}_{l}$ of the $l$-th path is independently generated according to the distribution $\bar{h}_{l} \propto g_{\mathcal{C}}\left(0, \sigma_{\bar{h}_{l}}^{2}\right)$ and $\sigma_{\bar{h}_{l}}^{2}=\exp (-0.1 l) /\left(\sum_{l} \sigma_{\bar{h}_{l}}^{2}\right)$. The normalized power delay profile (PDP) is shown in Table III. The total number of iterations and inner LDPC decoding iterations are $I=I_{\mathrm{in}} I_{\mathrm{out}}=50$ and $I_{c}=50$, respectively. Moreover, the spectral efficiency of SEFDM-IM signaling is calculated by $\eta=R_{c}\left(\log _{2}\left\lfloor\left(\begin{array}{l}N \\ K\end{array}\right)\right\rfloor+K \log _{2} M\right) /(\alpha N)$ bits/s/Hz [6].

We first evaluate the BER performance of the proposed equalizer $(I=1)$ and compare it to that of other existing equalizers conceived for SEFDM-IM signaling using known CSI, as shown in Fig. 4. Due to the high complexity of some of the existing methods, the number of subcarriers is set to $N_{s}=$ 16. The BML method $\left(L_{m}=4\right)$ in [28] and the MMSE-LLR method in [30] are extended to coded SEFDM-IM systems by calculating soft information representing the index bits and symbol bits. The BER performance of OFDM-IM signaling is also included as a benchmark. It is observed that the

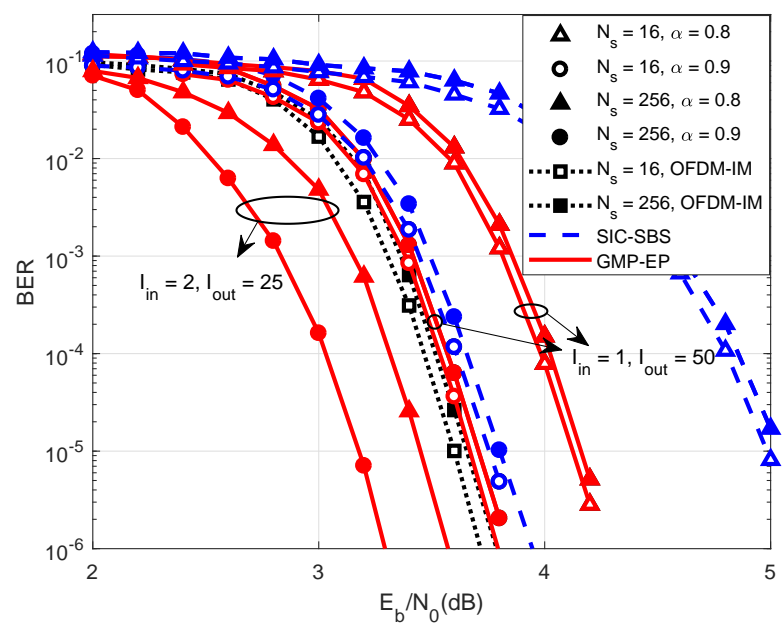

Fig. 5. BER performance of different equalizers for SEFDM-IM systems with known CSI, $(N, K)=(4,3)$.

BML equalizer and the MMSE-LLR equalizer do not perform well. This is because the BML equalizer ignores interferences from other IM subblocks and the MMSE-LLR equalizer only reserves two candidate active patterns for $K=1$. Compared to the OFDM-IM signaling, the MMSE-SBS equalizer suffers from $0.5 \mathrm{~dB}$ and $1.0 \mathrm{~dB}$ performance loss at $\mathrm{BER}=10^{-5}$ for $\alpha=0.9$ and $\alpha=0.8$, respectively. Due to joint interference cancellation and IM detection, the proposed hybrid GMP-EP equalizer attains a comparable BER performance at an $11 \%$ higher transmission rate than its orthogonal subcarrier based counterpart. When further decreasing the subcarrier packing factor, SEFDM-IM signaling improves the spectral efficiency by up to $25 \%$ at the cost of only $0.3 \mathrm{~dB}$ performance loss at $\mathrm{BER}=10^{-5}$.

In Fig. 5, we further evaluate the BER performance of the proposed equalizer $(I=50)$ and compare it to the SIC-SBS equalizer (which becomes the MMSE-SBS equalizer when $I=1)$. In the following simulations, the IM parameters are set to $(N, K)=(4,3)$. To illustrate the flexibility of the proposed equalizer, we provide simulation results for SEFDM-IM systems having various number of subcarriers, i.e., $N_{s}=16$ and $N_{s}=256$. It is observed that the BER of SEFDM-IM signaling only increases slightly upon increasing the number of subcarriers due to the ill-conditioning problem of SEFDMIM signaling. Compared to its OFDM-IM counterpart, for $\alpha=0.8$, SEFDM-IM signaling based on the proposed GMPEP equalizer achieves both $25 \%$ higher transmission rate and $0.2 \mathrm{~dB} \mathrm{E} \mathrm{b}_{\mathrm{b}} / \mathrm{N}_{0}$ gain at $\mathrm{BER}=10^{-5}$ by judiciously selecting the values of $I_{\text {in }}$ and $I_{\text {out }}$. By contrast, the SIC-SBS equalizer suffers from $1.4 \mathrm{~dB}$ performance loss for $\alpha=0.8$ due to the impact of severe equivalent interferences at the first iteration. 


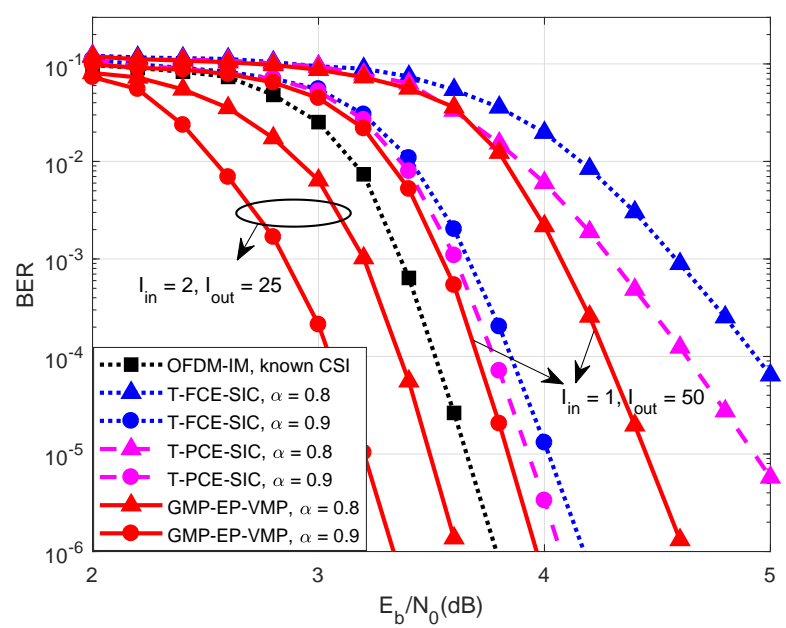

Fig. 6. BER performance of different joint channel estimation and equalization algorithms for SEFDM-IM systems.

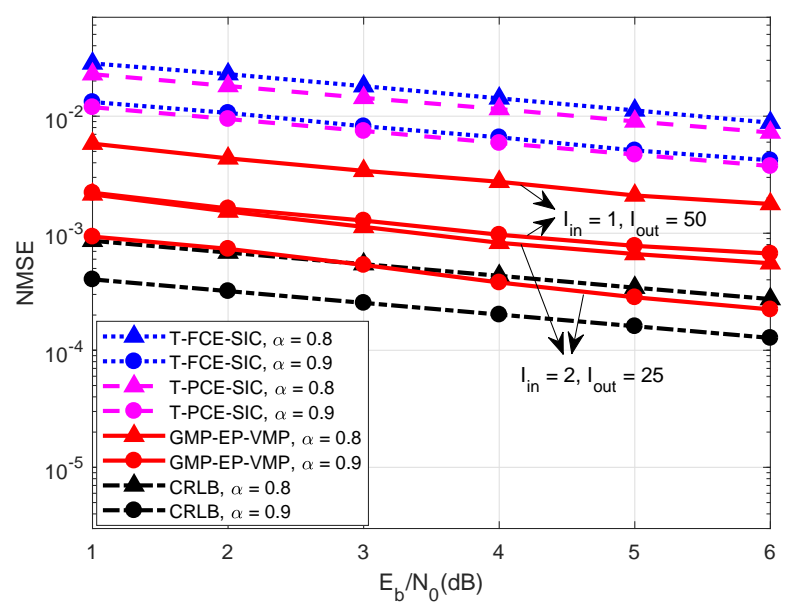

Fig. 7. NMSE performance of different joint channel estimation and equalization algorithms for SEFDM-IM systems.

As shown in Fig. 6 and Fig. 7, we evaluate the BER and NMSE performance of the proposed hybrid GMP-EP-VMP joint channel estimation and equalization method, respectively. The number of subcarriers is set to be $N_{s}=256$. The corresponding curves of the time-domain FCE-SIC and PCESIC algorithms, i.e., T-FCE-SIC and T-PCE-SIC, are also plotted for comparison. The BER performance of Nyquist signaling with perfect CSI is also included as a reference. As seen, the BER and NMSE performance of the T-PCESIC method are superior to those of the T-FCE-SIC method because the former only transmits pilots on mutually orthogonal subcarriers to alleviate the effects of the ill-conditioning problem of SEFDM signaling. Note that the proposed channel estimator employs not only the pilots, but also the transmitted symbols recovered in the previous iterations. Hence, the proposed method relying on the most appropriate activation order of the receive components outperforms other schemes in terms of their BER and NMSE performance, especially

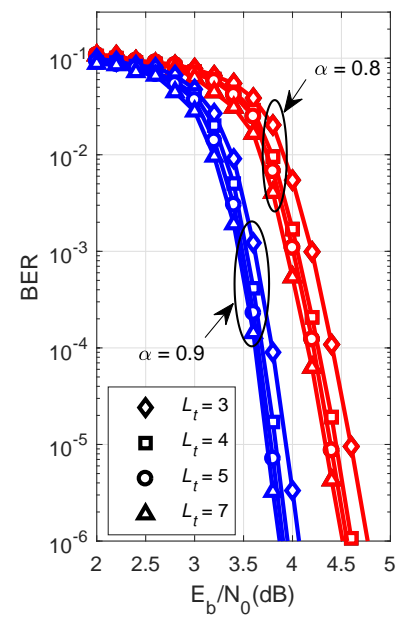

(a)

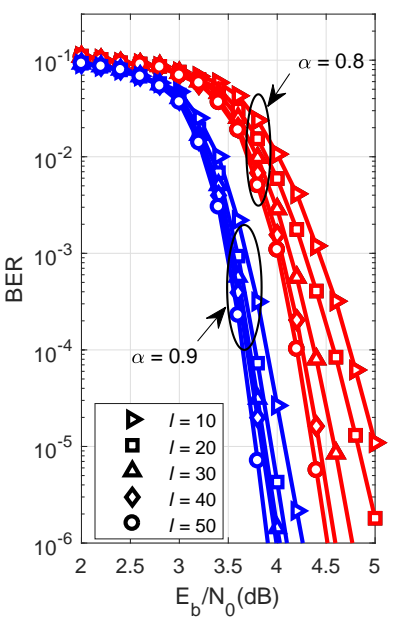

(b)
Fig. 8. Impact of the truncated length of interferences and the number of turbo iterations on BER performance $\left(I_{\mathrm{in}}=1\right)$.

for severe ICIs scenarios. Compared to that of OFDM-IM signaling, for $\alpha=0.9$, the $\mathrm{E}_{\mathrm{b}} / \mathrm{N}_{0}$ gain of the proposed GMPEP-VMP method using $I_{\text {in }}=2$ and $I_{\text {out }}=25$ is about $0.4 \mathrm{~dB}$ at BER $=10^{-5}$. Even if we further reduce the spacing of subcarriers to say $\alpha=0.8$, coded SEFDM-IM signaling still has about $0.2 \mathrm{~dB} \mathrm{E} \mathrm{E}_{\mathrm{b}} / \mathrm{N}_{0}$ gain. However, when the inner iteration of the equalizer runs only once, an additional $0.6 \mathrm{~dB}$ performance erosion may be encountered for $\alpha=0.8$ and $\alpha=0.9$. Hence, designing a judicious activation order is critical to the proposed methods. In Fig. 7, the CRLBs of the proposed channel estimator having various packing factors are included as benchmarks. The CRLB is derived under the assumption that $\tilde{L}$ transmitted SEFDM-IM symbols in the whole frame are known. In practice, only the first symbol contains pilot subcarriers and other symbols convey unknown transmitted data. Hence, the NMSE values of the proposed channel estimator are a little higher than the CRLBs.

The complexity of the proposed algorithm depends both on the length of the truncated interferences and on the number of turbo iterations. The impact of $L_{t}$ and $I$ on the BER performance is illustrated in Fig. 8. For simplicity, the number of subcarriers is $N_{s}=16$ in the following simulations. It is seen that the BER performance of SEFDM-IM signaling with various packing factors improves as the number $L_{t}$ or $I$ increases. When $L_{t}$ or $I$ is higher than a certain value, the performance gain becomes marginal, especially for dense subcarrier packing. In Fig. 8(a), significant performance gaps is observed between $L_{t}=3$ and $L_{t}=7$, which is due to the underestimation of the interferences induced by nonorthogonal signaling. By contrast, $L_{t} \geq 4$ is a reasonable approximation for $\alpha=0.8, \alpha=0.9$ in this case. In Fig. 8(b), the convergence speed of the proposed algorithm becomes slower upon reducing $\alpha$. The result coincides with the fact that a smaller $\alpha$ imposes stronger interferences. To strike a performance vs complexity balance, $I \geq 20$ is a reasonable option for $\alpha=0.8, \alpha=0.9$.

The BER performance of the proposed method with different combinations of the number of LDPC decoding iterations 


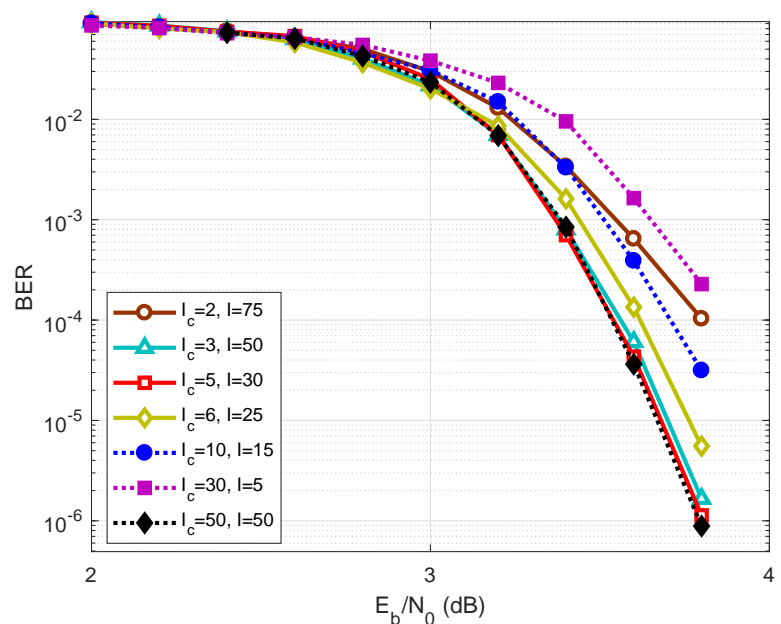

Fig. 9. BER performance of the proposed algorithm with different combinations of $I_{c}$ and $I\left(I_{\text {in }}=1\right)$.

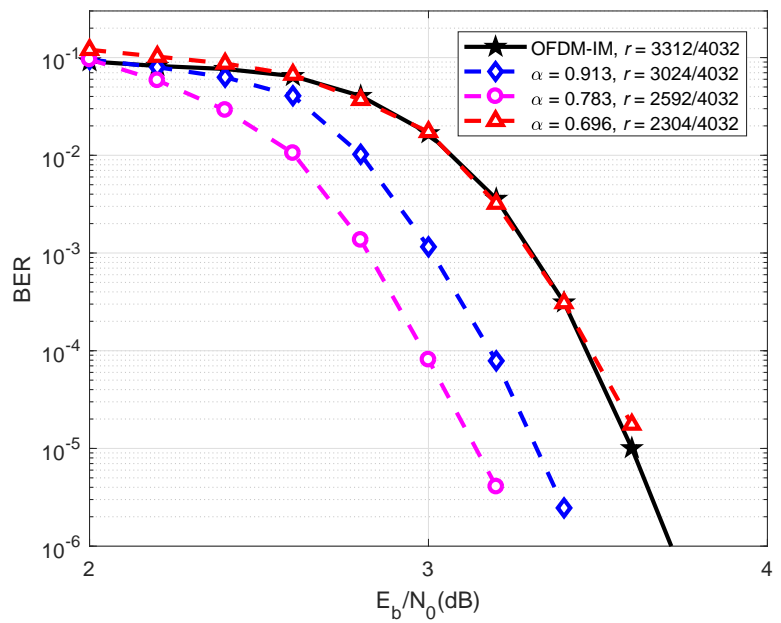

Fig. 10. BER performance of the proposed algorithm with different combinations of $\alpha$ and $r\left(I_{\text {in }}=1\right)$.

$I_{c}$ and turbo iterations $I$ are evaluated in Fig. 9, where $\alpha=0.9$. Since the complexity of LDPC decoding dominates the complexity of turbo receiver, we keep the total number of LDPC decoding, i.e., the product of $I_{c}$ and $I$, to be constant. It can be observed that, at the beginning, the BER performance of the proposed method improves with the increasing of $I_{c}$. Specifically, when $I_{c}=5, I=30$, the BER performance is very close to the one with $I_{c}=50, I=50$. However, when further increasing $I_{c}$, the BER performance degrades. This may due to the fact that the proposed method cannot coverage with inadequate turbo iterations. Therefore, message scheduling between LDPC decoding as well as channel estimation and equalization can be optimized to reduce the computational complexity.

In Fig. 10, we further evaluate the BER performance of SEFDM-IM signaling for a fixed spectral efficiency of $\eta=1.64 \mathrm{bits} / \mathrm{s} / \mathrm{Hz}$ at different combinations of the subcarrier packing factor $\alpha$ and code rate $r$, where $I_{c}=50$ and $I=50$.
In the simulations, we employ four LDPC codes having different code rates of $r=3312 / 4032, r=3024 / 4032$, $r=2592 / 4032, r=2304 / 4032$. The scenario of OFDMIM signaling with known CSI is plotted as the benchmark, where $\alpha=1$ and $r=3312 / 4032$. It is observed that the performance gain is about $0.3 \mathrm{~dB}$ for SEFDM-IM signaling associated with $\alpha=0.913, r=3024 / 4032$. Upon reducing the coding rate and the packing factor simultaneously to $\alpha=0.783, r=2592 / 4032$, the gain becomes $0.5 \mathrm{~dB}$ at the same bandwidth efficiency. Note that we cannot increase the performance gain by keep decreasing $r$ for a fixed $\eta$. When further reducing $\alpha$ and $r$, e.g., $\alpha=0.696, r=2304 / 4032$, the BER performance is degraded. This is due to the fact that when the subcarrier packing factor is much smaller than the Mazo limit [46], the residual ICI degrades the decoding performance. Hence, in practical applications of SEFDM-IM systems, we can strike a compromise between the packing factor and code rate.

\section{Conclusions}

A low-complexity joint channel estimation and equalization was conceived for SEFDM-IM signaling over frequencyselective fading channels. To extract the potential performance gain, we jointly performed interference mitigation and IM detection for the scenario of perfectly known CSI. By introducing the extended constellation concept and further reformulating the design problem via a linear state-space model, we constructed a Forney-style factor graph having two subgraphs. Based on Gaussian approximations of the discrete transmitted symbols via the EP method, we derived a lowcomplexity parametric message passing algorithm. For the scenario of unknown CSI, we constructed a multi-layer factor graph to tackle the problem of error propagation in channel estimation induced by the deactivated subcarriers of SEFDMIM signaling. To obtain Gaussian expressions for message passing between the channel estimator and equalizer, we built a soft node and derived the forward and backward messages according to VMP rules. Our simulation results showed that for a known CSI scenario, the proposed GMP-EP equalizer outperformed the extended non-iterative BML, MMSE-LLR, and MMSE-SBS methods. Furthermore, the performance of the proposed method was also superior to the iterative SICSBS method, especially for small packing factors. For an unknown CSI scenario, the proposed GMP-EP-VMP method performed very close to its known CSI counterpart. The complexity of the proposed GMP-EP equalizer increases linearly with the number of subcarriers $N_{s}$ for $L_{s} \ll N_{s}$, and that of the proposed GMP-EP-VMP channel estimation algorithm grows logarithmically with the length of the channel memory and linearly with the number of subcarriers.

\section{APPENDIX A}

DERIVATIONS OF (41) AND (42)

In contrast to the elementary or multiplication nodes, the extraordinary inner product node lacks explicit Gaussian message updating rules on FFG. Note that the inner product constraint is equivalent to a dot product of the corresponding 
independent vectors $\breve{\mathbf{h}}_{q}$ and $\breve{\mathbf{x}}_{q}$, while the inner elements are also assumed to be independent. According to BP rules [47], [48], we can compute the forward messages of $\breve{\mathbf{s}}_{q}$ element-byelement as

$$
\begin{aligned}
\vec{\mu}\left(s_{q}\right) & \propto \int \delta\left(s_{q}-h_{q} x_{q}\right) \vec{\mu}\left(x_{q}\right) \vec{\mu}\left(h_{q}\right) d x_{q} d h_{q} \\
& \propto \frac{1}{\left|h_{q}\right|} \int g_{\mathcal{C}}\left(\vec{m}_{x_{q}}^{\left(i_{t}\right)}, \vec{V}_{x_{q}}^{\left(i_{t}\right)} ; \frac{s_{q}}{h_{q}}\right) g_{\mathcal{C}}\left(\vec{m}_{h_{q}}^{\left(i_{t}\right)}, \vec{V}_{h_{q}}^{\left(i_{t}\right)} ; h_{q}\right) d h_{q} .
\end{aligned}
$$

However, it is hard to derive an analytical Gaussian expression for the above equation. To obtain the approximated Gaussian messages of $\breve{\mathbf{s}}_{q}$, according to the moment matching method [37], we have to calculate the first-order and secondorder moments of variables $\breve{\mathbf{s}}_{q}$. Based on the Mellin transform [49], it is easy to derive that the $n$ th-order moment of the product of two statistically independent random variables is equal to the product of two $n$ th-order moments of the corresponding independent random variables, i.e., we have $\mathbb{E}\left\{\left(x_{q} h_{q}\right)^{n}\right\}=\mathbb{E}\left\{x_{q}^{n}\right\} \mathbb{E}\left\{h_{q}^{n}\right\}$.

Based on the a priori probabilities obtained from the channel decoder and channel estimator, the approximated forward mean vector and covariance matrix of variables $\breve{\mathbf{s}}_{q}$ are given by

$$
\begin{aligned}
\overrightarrow{\mathbf{m}}_{\breve{\mathbf{s}}_{q}}^{\left(i_{t}\right)}= & \overrightarrow{\mathbf{m}}_{\dot{\mathbf{x}}_{q}}^{\left(i_{t}\right)} \odot \overrightarrow{\mathbf{m}}_{\breve{\mathbf{h}}_{q}}^{\left(i_{t}\right)} \\
\overrightarrow{\mathbf{V}}_{\breve{\mathbf{s}}_{q}}^{\left(i_{t}\right)}= & {\left[\mathcal{D}\left(\overrightarrow{\mathbf{m}}_{\dot{\mathbf{x}}_{q}}^{\left(i_{t}\right)} \odot \overrightarrow{\mathbf{m}}_{\dot{\mathbf{x}}_{q}}^{\left(i_{t}\right)}\right)+\overrightarrow{\mathbf{V}}_{\breve{\mathbf{x}}_{q}}^{\left(i_{t}\right)}\right] } \\
& \times\left[\mathcal{D}\left(\overrightarrow{\mathbf{m}}_{\breve{\mathbf{h}}_{q}}^{\left(i_{t}\right)} \odot \overrightarrow{\mathbf{m}}_{\breve{\mathbf{h}}_{q}}^{\left(i_{t}\right)}\right)+\overrightarrow{\mathbf{V}}_{\breve{\mathbf{h}}_{q}}^{\left(i_{t}\right)}\right]-\mathcal{D}\left(\overrightarrow{\mathbf{m}}_{\breve{\mathbf{s}}_{q}}^{\left(i_{t}\right)} \odot \overrightarrow{\mathbf{m}}_{\breve{\mathbf{s}}_{q}}^{\left(i_{t}\right)}\right)
\end{aligned}
$$

Hence, the forward mean and variance of the variable $s_{k}$ forwarded from the channel estimator to the equalizer is represented by (41) and (42).

\section{APPENDiX B}

DERIVATIONS OF (44) AND (45)

According to the VMP rules [43], the backward message of variables $\breve{\mathbf{h}_{q}^{l}}$ is rewritten as

$$
\overleftarrow{\mu}\left(\breve{\mathbf{h}}_{q}^{\tilde{l}}\right) \propto \exp \left(\int \ln f_{q}^{\tilde{l}}\left(\breve{\mathbf{x}}_{q}^{\tilde{l}}, \breve{\mathbf{h}}_{q}^{\tilde{l}}\right) b\left(\breve{\mathbf{x}}_{q}^{\tilde{l}}\right) d \breve{\mathbf{x}}_{q}^{\tilde{l}}\right)
$$

where the $a$ posteriori belief obeys $b\left(\breve{\mathbf{x}}_{q}^{\tilde{l}}\right) \propto$ $g_{\mathcal{C}}\left(\mathbf{m}_{\widetilde{\mathbf{x}}_{q}^{\tilde{I}}}^{\left(i_{t}\right)}, \mathbf{V}_{\breve{\mathbf{x}}_{q}^{\tilde{\tau}}}^{\left(i_{t}\right)} ; \breve{\mathbf{x}}_{q}^{\tilde{l}}\right)$. Next, the backward message of variables $\breve{\mathbf{h}}_{q}^{\tilde{l}}$ at the $\left(i_{t}\right)$-th iteration is derived as

$$
\begin{aligned}
& \overleftarrow{\mu}\left(\breve{\mathbf{h}}_{q}^{\tilde{l}}\right) \propto \exp \left[-\int\left(\mathcal{D}\left(\breve{\mathbf{x}}_{q}^{\tilde{l}}\right) \breve{\mathbf{h}}_{q}^{\tilde{l}}-\overleftarrow{\mathbf{m}}_{\breve{\mathbf{s}}_{q}^{\tilde{l}}}^{\left(i_{t}\right)}\right)^{H}\left(\overleftarrow{\mathbf{V}}_{\breve{\mathbf{s}}_{q}^{\tilde{\tau}}}^{\left(i_{t}\right)}\right)^{-1}\left(\mathcal{D}\left(\breve{\mathbf{x}}_{q}^{\tilde{l}}\right) \breve{\mathbf{h}}_{q}^{\tilde{l}}-\overleftarrow{\mathbf{m}}_{\widetilde{\mathbf{s}}_{q}^{\tilde{\tau}}}^{\left(i_{t}\right)}\right)\right. \\
& \left.\times g_{\mathcal{C}}\left(\mathbf{m}_{\breve{\mathbf{x}}_{q}^{\tilde{l}}}^{\left(i_{t}\right)}, \mathbf{V}_{\breve{\mathbf{x}}_{q}^{\tilde{\tau}}}^{\left(i_{i^{\prime}}\right)} ; \breve{\mathbf{x}}_{q}^{\tilde{l}}\right) d \breve{\mathbf{x}}_{q}^{\tilde{l}}\right] \\
& \propto \exp \left[-\left(\breve{\mathbf{h}}_{q}^{\tilde{l}}\right)^{H}\left(\int \mathcal{D}\left(\breve{\mathbf{x}}_{q}^{\tilde{l}}\right)^{H}\left(\overleftarrow{\mathbf{V}}_{\breve{\mathbf{s}}_{q}^{\tilde{\tau}}}^{\left(i_{t}\right)}\right)^{-1} \boldsymbol{\Lambda}_{\breve{\mathbf{x}}_{q}^{\tilde{l}}}\right.\right. \\
& \left.\times g_{\mathcal{C}}\left(\mathbf{m}_{\widetilde{\mathbf{x}}_{q}^{\tilde{L}}}^{\left(i_{t}\right)}, \mathbf{V}_{\widetilde{\mathbf{x}}_{q}^{\tilde{l}}}^{\left(i_{t}\right)} ; \breve{\mathbf{x}}_{q}^{\tilde{l}}\right) d \breve{\mathbf{x}}_{q}^{\tilde{l}}\right) \breve{\mathbf{h}}_{q}^{\tilde{l}} \\
& +2 \operatorname{Re}\left\{\left(\breve{\mathbf{h}}_{q}^{\tilde{l}}\right)^{H}\left(\int \boldsymbol{\Lambda}_{\breve{\mathbf{x}}_{q}^{\tilde{\tau}}}^{H} g_{\mathcal{C}}\left(\mathbf{m}_{\breve{\mathbf{x}}_{q}^{\tilde{\tau}}}^{\left(i_{t}\right)}, \mathbf{V}_{\breve{\mathbf{x}}_{q}^{\tilde{\tau}}}^{\left(i_{t}\right)} ; \breve{\mathbf{x}}_{q}^{\tilde{l}}\right) d \breve{\mathbf{x}}_{q}^{\tilde{l}}\right)\right. \\
& \left.\left.\times\left(\overleftarrow{\mathbf{V}}_{\widetilde{\mathbf{s}}_{q}^{\tilde{\tau}}}^{\left(i_{t}\right)}\right)^{-1} \overleftarrow{\mathbf{m}}_{\widetilde{\mathbf{s}}_{q}^{\tau}}^{\left(i_{t}\right)}\right\}\right] \\
& \propto \exp \left[-\left(\breve{\mathbf{h}}_{q}^{\tilde{l}}\right)^{H} \mathcal{D}\left(\mathbb{E}\left\{\left(\breve{\mathbf{x}}_{q}^{\tilde{l}}\right)^{H} \odot \breve{\mathbf{x}}_{q}^{\tilde{l}}\right\}\right)\left(\overleftarrow{\mathbf{V}}_{\breve{\mathbf{s}}_{q}^{\tilde{\tau}}}^{\left(i_{t}\right)}\right)^{-1} \breve{\mathbf{h}}_{q}^{\tilde{l}}\right. \\
& \left.+2 \operatorname{Re}\left\{\left(\breve{\mathbf{h}}_{q}^{\tilde{l}}\right)^{H} \mathcal{D}\left(\mathbb{E}\left\{\left(\breve{\mathbf{x}}_{q}^{\tilde{l}}\right)^{H}\right\}\right)\left(\overleftarrow{\mathbf{V}}_{\breve{\mathbf{s}}_{q}^{\tilde{\tau}}}^{\left(i_{t}\right)}\right)^{-1} \overleftarrow{\mathbf{m}}_{\widetilde{\mathbf{s}}_{q}^{\tilde{\tau}}}^{\left(i_{t}\right)}\right\}\right] \\
& \propto \exp \left[-\left(\breve{\mathbf{h}}_{q}^{\tilde{l}}\right)^{H}\left(\mathbf{V}_{\widetilde{\mathbf{x}}_{q}^{\tilde{l}}}^{\left(i_{t}\right)}+\mathcal{D}\left(\left(\mathbf{m}_{\breve{\mathbf{x}}_{q}^{\tilde{l}}}^{\left(i_{t}\right)}\right)^{H} \odot \mathbf{m}_{\widetilde{\mathbf{x}}_{q}^{\tilde{\tau}}}^{\left(i_{t}\right)}\right)\right)\left(\overleftarrow{\mathbf{V}}_{\breve{\mathbf{s}}_{q}^{\tilde{\tau}}}^{\left(i_{t}\right)}\right)^{-1} \breve{\mathbf{h}}_{q}^{\tilde{l}}\right. \\
& \left.+2 \operatorname{Re}\left\{-\left(\breve{\mathbf{h}}_{q}^{\tilde{l}}\right)^{H} \mathcal{D}\left(\left(\mathbf{m}_{\widetilde{\mathbf{x}}_{q}^{\tilde{\tau}}}^{\left(i_{t}\right)}\right)^{H} \odot \overleftarrow{\mathbf{m}}_{\widetilde{\mathbf{s}}_{q}^{\tilde{\tau}}}^{\left(i_{t}\right)}\right)\left(\overleftarrow{\mathbf{V}}_{\widetilde{\mathbf{s}}_{q}^{\tilde{\tau}}}^{\left(i_{t}\right)}\right)^{-1}\right\}\right]
\end{aligned}
$$

\section{APPENDIX C}

\section{CRLB FOR THE PROPOSED CHANNEL ESTIMATOR}

First of all, we should prove that the proposed channel estimator is unbiased. Assuming that the unknown CIR vector $\overline{\mathbf{h}}=\left[\bar{h}_{0}, \cdots, \bar{h}_{L-1}\right]^{T}$ remains unchanged during $\tilde{L}$ SEFDMIM symbols, the $\tilde{l}$-th received SEFDM-IM symbol in (3) can be rewritten as $\mathbf{r}_{\tilde{l}}=\boldsymbol{\Phi} \mathbf{X}_{\tilde{l}} \boldsymbol{\Theta} \overline{\mathbf{h}}+\boldsymbol{\omega}_{\tilde{l}}$, where $\boldsymbol{\Theta}$ is a $N_{s} \times L$ matrix and the element on the $n$-th row and the $l$-th column is $\theta_{n, l}=e^{-\frac{j 2 \pi \alpha n T_{l}}{T_{s}}}$. Based on Bayesian rules, the MAP estimator of the unknown channel parameters is derived as

$$
\hat{\overline{\mathbf{h}}}=\arg \max _{\overline{\mathbf{h}}} \sum_{\tilde{l}}\left(\ln p\left(\mathbf{r}_{\tilde{l}} \mid \overline{\mathbf{h}}, \mathbf{X}_{\tilde{l}}\right)+\ln p\left(\mathbf{X}_{\tilde{l}}\right)+\ln p(\overline{\mathbf{h}})\right),
$$

where $p\left(\mathbf{r}_{\tilde{l}} \mid \overline{\mathbf{h}}, \mathbf{X}_{\tilde{l}}\right)=g_{\mathcal{C}}\left(\boldsymbol{\Phi} \mathbf{X}_{\tilde{l}} \boldsymbol{\Theta} \overline{\mathbf{h}}, \mathbf{V} ; \mathbf{r}_{\tilde{l}}\right)$ and $\mathbf{V}=\sigma_{\omega}^{2} \boldsymbol{\Phi}$. Here, the a priori probability $p(\overline{\mathbf{h}})$ of the CIRs describes the accuracy of channel initialization. Hence, we assume that the a priori probability of the CIRs obeys the Gaussian distribution, i.e., $p(\overline{\mathbf{h}})=g_{\mathcal{C}}\left(\overline{\mathbf{h}}^{0}, \mathbf{V}_{\overline{\mathbf{h}}^{0}} ; \overline{\mathbf{h}}\right)$, where the mean vector is initialized as $\overline{\mathbf{h}}^{0}=\left[\left(\boldsymbol{\Phi} \mathbf{X}_{0} \boldsymbol{\Theta}\right)^{H} \mathbf{\Phi} \mathbf{X}_{0} \boldsymbol{\Theta}\right]^{-1}\left(\boldsymbol{\Phi} \mathbf{X}_{0} \boldsymbol{\Theta}\right)^{H} \mathbf{r}_{0}$ using pilot-based $\mathrm{ZF}$ method in [20], the covariance matrix is initialized as $\mathbf{V}_{\overline{\mathbf{h}}^{0}}=\mathcal{D}\left(\boldsymbol{\sigma}^{0}\right)$ and the $l$-th element of $\boldsymbol{\sigma}^{0}$ is $\sigma_{l}^{0}=\sigma_{\bar{h}_{l}}^{2}-\left|\bar{h}_{l}\right|^{2}$. The first-order partial derivative of $\ln p\left(\overline{\mathbf{h}} \mid \mathbf{r}_{0}, \cdots, \mathbf{r}_{\tilde{L}-1}, \mathbf{X}_{0}, \cdots, \mathbf{X}_{\tilde{L}-1}\right)$ is

$$
\begin{aligned}
& \frac{\partial \ln p\left(\overline{\mathbf{h}} \mid \mathbf{r}_{0}, \cdots, \mathbf{r}_{\tilde{L}-1}, \mathbf{X}_{0}, \cdots, \mathbf{X}_{\tilde{L}-1}\right)}{\partial \overline{\mathbf{h}}} \\
& =\sum_{\tilde{l}} \frac{\partial \ln p\left(\mathbf{r}_{\tilde{l}} \overline{\mathbf{h}}, \mathbf{X}_{\tilde{l}}\right)}{\partial \overline{\mathbf{h}}}+\frac{\partial \ln p(\overline{\mathbf{h}})}{\partial \overline{\mathbf{h}}} \\
& =-\sum_{\tilde{l}}\left(\boldsymbol{\Phi} \mathbf{X}_{\tilde{l}} \boldsymbol{\Theta}\right)^{H} \mathbf{V}^{-1}\left(\mathbf{r}_{\tilde{l}}-\mathbf{\Phi} \mathbf{X}_{\tilde{l}} \boldsymbol{\Theta} \overline{\mathbf{h}}\right)+\mathbf{V}_{\overline{\mathbf{h}}^{0}}^{-1}\left(\overline{\mathbf{h}}-\overline{\mathbf{h}}^{0}\right),
\end{aligned}
$$

and setting it to zero yields $\hat{\overline{\mathbf{h}}}=\left(\sum_{\tilde{l}}\left(\boldsymbol{\Phi} \mathbf{X}_{\tilde{l}} \boldsymbol{\Theta}\right)^{H} \mathbf{V}^{-1} \mathbf{\Phi} \mathbf{X}_{\tilde{l}} \boldsymbol{\Theta}+\right.$ $\left.\mathbf{V}_{\overline{\mathbf{h}}^{0}}^{-1}\right)^{-1}\left(\sum_{\tilde{l}}\left(\mathbf{\Phi} \mathbf{X}_{\tilde{l}} \mathbf{\Theta}\right)^{H} \mathbf{V}^{-1} \mathbf{r}_{\tilde{l}}+\mathbf{V}_{\overline{\mathbf{h}}^{0}}^{-1} \overline{\mathbf{h}}^{0}\right)$. It is easy to prove 
that $\mathbb{E}\{\hat{\overline{\mathbf{h}}}\}=\overline{\mathbf{h}}$. Hence, the proposed channel estimator is unbiased.

According to (70), the expectation of the first-order partial derivative of the joint probability distribution function (PDF) equals zero, i.e. the $p\left(\mathbf{r}_{0}, \cdots, \mathbf{r}_{\tilde{L}-1}, \mathbf{X}_{0}, \cdots, \mathbf{X}_{\tilde{L}-1}, \overline{\mathbf{h}}\right)$ satisfies the regularity condition. Then, the covariance matrix of the proposed unbiased estimator $\hat{\overline{\mathbf{h}}}$ satisfies $\mathbf{C}_{\overline{\mathbf{h}}} \geq \mathbf{I}^{-1}(\overline{\mathbf{h}})$. According to the derivations in [50], the Fisher information matrix $\mathbf{I}(\overline{\mathbf{h}})$ is

$$
\mathbf{I}(\overline{\mathbf{h}})=\sum_{\tilde{l}}\left(\boldsymbol{\Phi} \mathbf{X}_{\tilde{l}} \boldsymbol{\Theta}\right)^{H} \mathbf{V}^{-1} \mathbf{\Phi} \mathbf{X}_{\tilde{l}} \boldsymbol{\Theta} .
$$

Therefore, the CRLB for the proposed channel estimator is $\mathrm{CRLB}=\operatorname{tr}\left[\mathbf{I}^{-1}(\overline{\mathbf{h}})\right]$.

\section{REFERENCES}

[1] V. W. S. Wong, R. Schober, D. W. K. Ng, and L.-C. Wang, Key Technologies for $5 G$ Wireless Systems. Cambridge University Press, 2017.

[2] L. Dai, B. Wang, Z. Ding, Z. Wang, S. Chen, and L. Hanzo, "A survey of non-orthogonal multiple access for 5G," IEEE Commun. Surveys Tuts., vol. 20, no. 3, pp. 2294-2323, 3rd Quart. 2018.

[3] Q. Shi, N. Wu, X. Ma, and H. Wang, "Frequency-domain joint channel estimation and decoding for faster-than-Nyquist signaling," IEEE Trans. Commun., vol. 66, no. 2, pp. 781-795, Feb. 2018.

[4] J. Zhang, E. Björnson, M. Matthaiou, D. W. K. Ng, H. Yang, and D. J. Love, "Prospective multiple antenna technologies for beyond 5G," arXiv preprint, arXiv:1910.00092, 2019.

[5] Y. Liu, Z. Qin, M. Elkashlan, Z. Ding, A. Nallanathan, and L. Hanzo, "Nonorthogonal multiple access for 5G and beyond," Proc. IEEE, vol. 105, no. 12, pp. 2347-2381, Dec. 2017.

[6] P. Banelli, S. Buzzi, G. Colavolpe, A. Modenini, F. Rusek, and A. Ugolini, "Modulation formats and waveforms for 5G networks: Who will be the heir of OFDM?: An overview of alternative modulation schemes for improved spectral efficiency," IEEE Signal Process. Mag., vol. 31, no. 6, pp. 80-93, Nov. 2014.

[7] G. Wunder, P. Jung, M. Kasparick, T. Wild, F. Schaich, Y. Chen, S. T. Brink, I. Gaspar, N. Michailow, A. Festag, L. Mendes, N. Cassiau, D. Ktenas, M. Dryjanski, S. Pietrzyk, B. Eged, P. Vago, and F. Wiedmann, "5GNOW: Non-orthogonal, asynchronous waveforms for future mobile applications," IEEE Commun. Mag., vol. 52, no. 2, pp. 97-105, Feb. 2014.

[8] N. Michailow, M. Matthé, I. S. Gaspar, A. N. Caldevilla, L. L. Mendes, A. Festag, and G. Fettweis, "Generalized frequency division multiplexing for 5th generation cellular networks," IEEE Trans. Commun., vol. 62, no. 9, pp. 3045-3061, 2014.

[9] X. Chen, D. W. K. Ng, W. Yu, E. G. Larsson, N. Al-Dhahir, and R. Schober, "Massive access for $5 \mathrm{G}$ and beyond," arXiv preprint, arXiv:2002.03491, 2020.

[10] M. Jia, Z. Yin, Q. Guo, G. Liu, and X. Gu, "Downlink design for spectrum efficient IoT network," IEEE Internet Things J., vol. 5, no. 5, pp. 3397-3404, Oct. 2018.

[11] T. $\mathrm{Xu}$ and I. Darwazeh, "Transmission experiment of bandwidth compressed carrier aggregation in a realistic fading channel," IEEE Trans. Veh. Technol., vol. 66, no. 5, pp. 4087-4097, May 2017.

[12] I. Darwazeh, T. Xu, T. Gui, Y. Bao, and Z. Li, "Optical SEFDM system; bandwidth saving using non-orthogonal sub-carriers," IEEE Photon. Technol. Lett., vol. 26, no. 4, pp. 352-355, Feb. 2014.

[13] Y. Wang, Y. Zhou, T. Gui, K. Zhong, X. Zhou, L. Wang, A. P. T. Lau, C. Lu, and N. Chi, "Efficient MMSE-SQRD based MIMO decoder for SEFDM based 2.4-Gb/s-spectrum-compressed WDM VLC system," IEEE Photon. J., vol. 8, no. 4, pp. 1-9, Aug. 2016.

[14] H. Ghannam and I. Darwazeh, "SEFDM over satellite systems with advanced interference cancellation," IET Commun., vol. 12, no. 1, pp. 59-66, 2018.

[15] I. Kanaras, A. Chorti, M. R. D. Rodrigues, and I. Darwazeh, "A fast constrained sphere decoder for ill conditioned communication systems," IEEE Commun. Lett., vol. 14, no. 11, pp. 999-1001, Nov. 2010.

[16] S. Isam, I. Kanaras, and I. Darwazeh, "A truncated SVD approach for fixed complexity spectrally efficient FDM receivers," in Proc. IEEE Wireless Commun. Netw. Conf., Mar. 2011, pp. 1584-1589.
[17] T. Xu, R. C. Grammenos, F. Marvasti, and I. Darwazeh, "An improved fixed sphere decoder employing soft decision for the detection of nonorthogonal signals," IEEE Commun. Lett., vol. 17, no. 10, pp. 19641967, Oct. 2013.

[18] T. Xu and I. Darwazeh, "A soft detector for spectrally efficient systems with non-orthogonal overlapped sub-carriers," IEEE Commun. Lett., vol. 18 , no. 10 , pp. 1847-1850, Oct. 2014.

[19] B. Yu, H. Zhang, X. Hong, and C. Guo, "Channel equalization and data detection for SEFDM over frequency selective fading channels," IET Commun., vol. 12, no. 18, pp. 2315-2323, Nov. 2018.

[20] A. Chorti, I. Kanaras, M. R. D. Rodrigues, and I. Darwazeh, "Joint channel equalization and detection of spectrally efficient FDM signals," in Proc. IEEE Int. Symp. Pers., Indoor Mobile Radio Commun., Sep. 2010, pp. 177-182.

[21] S. Isam and I. Darwazeh, "Robust channel estimation for spectrally efficient FDM system," in Proc. 19th Int. Conf. Telecommun., Apr. 2012, pp. 1-6.

[22] H. Ghannam and I. Darwazeh, "Robust channel estimation methods for spectrally efficient FDM systems," in Proc. IEEE 87th Veh. Technol. Conf. (Spring), Jun. 2018, pp. 1-6.

[23] N. Ishikawa, S. Sugiura, and L. Hanzo, "50 years of permutation, spatial and index modulation: From classic RF to visible light communications and data storage," IEEE Commun. Surveys Tuts., vol. 20, no. 3, pp. 1905-1938, 3rd Quart. 2018.

[24] N. Ishikawa, S. Sugiura and L. Hanzo, "Subcarrier-index modulation aided OFDM - will it work?" IEEE Access, vol. 4, pp. 2580-2593, 2016.

[25] E. Başar, Ü. Aygölü, E. Panayırcı, and H. V. Poor, "Orthogonal frequency division multiplexing with index modulation," IEEE Trans. Signal Process., vol. 61, no. 22, pp. 5536-5549, Nov. 2013.

[26] M. Wen, X. Cheng, L. Yang, Y. Li, X. Cheng, and F. Ji, "Index modulated OFDM for underwater acoustic communications," IEEE Commun. Mag., vol. 54, no. 5, pp. 132-137, May 2016.

[27] T. Mao, W. Qi, Z. Wang, and C. Sheng, "Novel index modulation techniques: A survey," IEEE Commun. Surveys Tuts., vol. 21, no. 1, pp. 315-348, 2019.

[28] H. Liu, L. Liu, and P. Wang, "Spectrally efficient nonorthogonal frequency division multiplexing with index modulation," in Proc. 17th Int. Conf. Parallel Distrib. Comput. Appl. Technol., Dec. 2016, pp. 290-293.

[29] T. Ishihara and S. Sugiura, "Faster-than-Nyquist signaling with index modulation," IEEE Wireless Commun. Lett., vol. 6, no. 5, pp. 630-633, Oct. 2017.

[30] M. Nakao and S. Sugiura, "Spectrally efficient frequency division multiplexing with index-modulated non-orthogonal subcarriers," IEEE Wireless Commun. Lett., vol. 8, no. 1, pp. 233-236, Feb. 2019.

[31] T. van Erven and P. Harremos, "Rényi divergence and Kullback-Leibler divergence," IEEE Trans. Inf. Theory, vol. 60, no. 7, pp. 3797-3820.

[32] S. Isam and I. Darwazeh, "Simple DSP-IDFT techniques for generating spectrally efficient FDM signals," in Proc. 7th Int. Symp. Commun. Syst. Netw. Digital Signal Process., Jul. 2010, pp. 20-24.

[33] S. I. A. Ahmed, "Spectrally efficient FDM communication signals and transceivers: Design, mathematical modelling and system optimization," $\mathrm{Ph}$.D. dissertation, University College London (University of London), 2011.

[34] I. Sekita, T. Kurita, and N. Otsu, "Complex autoregressive model for shape recognition," IEEE Trans. Pattern Anal. Machine Intell., vol. 14, no. 4, pp. 489-496, Apr. 1992.

[35] H. Loeliger, J. Dauwels, J. Hu, S. Korl, L. Ping, and F. R. Kschischang, "The factor graph approach to model-based signal processing," Proc. IEEE, vol. 95, no. 6, pp. 1295-1322, Jun. 2007.

[36] Q. Guo and L. Ping, "LMMSE turbo equalization based on factor graphs," IEEE J. Sel. Areas Commun., vol. 26, no. 2, pp. 311-319, Feb. 2008.

[37] J. Céspedes, P. M. Olmos, M. Sánchez-Fernández, and F. Perez-Cruz, "Expectation propagation detection for high-order high-dimensional MIMO systems," IEEE Trans. Commun., vol. 62, no. 8, pp. 2840-2849, Aug. 2014.

[38] W. Yuan, N. Wu, Q. Guo, Y. Li, C. Xing, and J. Kuang, "Iterative receivers for downlink MIMO-SCMA: Message passing and distributed cooperative detection," IEEE Trans. Wireless Commun., vol. 17, no. 5, pp. 3444-3458, May 2018.

[39] M. Senst and G. Ascheid, "How the framework of expectation propagation yields an iterative IC-LMMSE MIMO receiver,' in Proc. IEEE Global Telecommun. Conf., Dec. 2011, pp. 1-6.

[40] N. Wu, W. Yuan, Q. Guo, and J. Kuang, "A hybrid BP-EP-VMP approach to joint channel estimation and decoding for FTN signaling 
over frequency selective fading channels," IEEE Access, vol. 5, pp. 6849-6858, 2017.

[41] Z. Hu, F. Chen, M. Wen, F. Ji, and H. Yu, "Low-complexity LLR calculation for OFDM with index modulation," IEEE Wireless Commun. Lett., vol. 7, no. 4, pp. 618-621, Aug. 2018.

[42] J. Winn and C. M. Bishop, "Variational message passing," J. Mach. Learn. Res., vol. 6, pp. 661-694, Apr. 2005.

[43] J. Dauwels, "On variational message passing on factor graphs," in Proc. IEEE Int. Symp. Inf. Theory, Jun. 2007, pp. 2546-2550.

[44] A. Barbieri, D. Fertonani, and G. Colavolpe, "Time-frequency packing for linear modulations: Spectral efficiency and practical detection schemes," IEEE Trans. Commun., vol. 57, no. 10, pp. 2951-2959, Oct. 2009.

[45] E. Panayirci, M. T. Altabbaa, M. Uysal, and H. V. Poor, "Sparse channel estimation for OFDM-based underwater acoustic systems in Rician fading with a new OMP-MAP algorithm," IEEE Trans. Signal Process., vol. 67, no. 6, pp. 1550-1565, Mar. 2019.

[46] J. E. Mazo, "Faster-than-Nyquist signaling," The Bell Syst. Technical J., vol. 54, no. 8, pp. 1451-1462, Oct. 1975.

[47] W. Yuan, N. Wu, A. Zhang, X. Huang, Y. Li, and L. Hanzo, "Iterative receiver design for FTN signaling aided sparse code multiple access," IEEE Trans. Wireless Commun., vol. 19, no. 2, pp. 915-928, Feb. 2020.

[48] W. Yuan, N. Wu, Q. Guo, D. W. K. Ng, J. Yuan, and L. Hanzo, "Iterative joint channel estimation, user activity tracking, and data detection for FTN-NOMA systems supporting random access," IEEE Trans. Commun., vol. 68, no. 5, pp. 2963-2977, Feb. 2020.

[49] A. M. Mathai, A handbook of generalized special functions for statistical and physical sciences. Oxford University Press, USA, 1993.

[50] S. M. Kay, Fundamentals of Statistical Signal Processing. Upper Saddle River, NJ: Prentice-Hall, 2017.

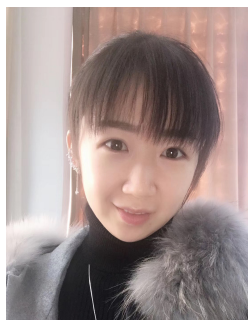

Yunsi Ma (S'19) received the B.S. degree from Nanjing University of Aeronautics and Astronautics, Nanjing, China, in 2013, and the M.S. degree from China Academy of Space Technology, Beijing, China, in 2016. She is currently working toward the $\mathrm{Ph} . \mathrm{D}$. degree with the School of Information and Electronics, Beijing Institute of Technology, Beijing, China. Her research interests include statistical inference on graphical models and its application to wireless communications.

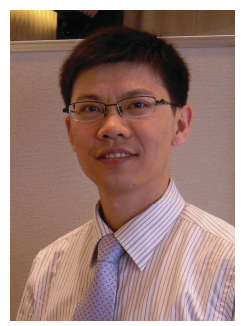

Nan Wu (M'11) received his B.S., M.S. and Ph.D. degrees from Beijing Institute of Technology (BIT), Beijing, China in 2003, 2005 and 2011, respectively. From 2008 to 2009, he was a Visiting Ph.D. Student with the Department of Electrical Engineering, Pennsylvania State University, USA. He is currently a Professor with the School of Information and Electronics, BIT. His research interests include signal processing in wireless communication networks. He was a recipient of the National Excellent Doctoral Dissertation Award by MOE of China in 2013. He serves as an Editorial Board Member of the IEEE Wireless Communications Letters, the IEEE Access, the International Journal of Electronics and Communications, and the KSII Transactions on Internet and Information Systems.

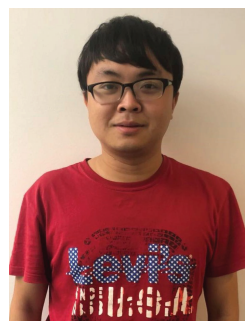

Weijie Yuan (S'15, M'20) received the Ph.D. degree from the Beijing Institute of Technology, China, and the Ph.D. degree from the University of Technology Sydney, Australia, in 2019. He is currently a Research Associate with the School of Electrical Engineering and Telecommunications, University of New South Wales, Sydney, Australia. He has served as a Research Assistant with the University of Sydney, a Visiting Associate Fellow with the University of Wollongong, and a Visiting Fellow with the University of Southampton, from 2017 to 2019. In 2016, he was a Visiting Ph.D. Student with the Institute of Telecommunications, Vienna University of Technology, Austria. His research interest includes statistical inference on graphical models. He has served as a TPC member for several conferences.

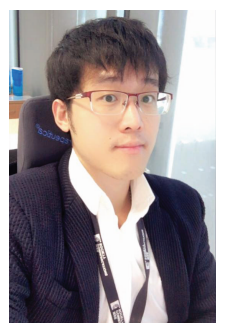

Derrick Wing Kwan Ng (S'06-M'12-SM'17) received the bachelor degree with first-class honors and the Master of Philosophy (M.Phil.) degree in electronic engineering from the Hong Kong University of Science and Technology (HKUST) in 2006 and 2008, respectively. He received his Ph.D. degree from the University of British Columbia (UBC) in 2012. He was a senior postdoctoral fellow at the Institute for Digital Communications, Friedrich-Alexander-University Erlangen-Nürnberg (FAU), Germany. He is now working as a Senior Lecturer and a Scientia Fellow at the University of New South Wales, Sydney, Australia. His research interests include convex and non-convex optimization, physical layer security, wireless information and power transfer, and green (energy-efficient) wireless communications. Dr. Ng received the Best Paper Awards at the IEEE TCGCC Best Journal Paper Award 2018, INISCOM 2018, IEEE International Conference on Communications (ICC) 2018, IEEE International Conference on Computing, Networking and Communications (ICNC) 2016, IEEE Wireless Communications and Networking Conference (WCNC) 2012, the IEEE Global Telecommunication Conference (Globecom) 2011, and the IEEE Third International Conference on Communications and Networking in China 2008. He has been serving as an editorial assistant to the Editor-in-Chief of the IEEE Transactions on Communications from Jan. 2012 to Dec. 2019. In addition, he is listed as a Highly Cited Researcher by Clarivate Analytics in 2018 and 2019.

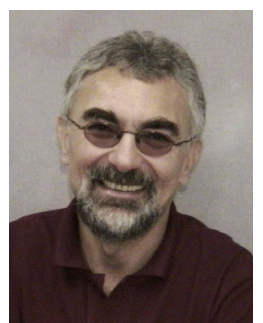

Lajos Hanzo (http://www-mobile.ecs.soton.ac.uk, https://en.wikipedia.org/wiki/Lajos_Hanzo) (FIEEE'04, Fellow of the Royal Academy of Engineering (FREng), of the IET and of EURASIP), received his Master degree and Doctorate in 1976 and 1983, respectively from the Technical University (TU) of Budapest. He was also awarded the Doctor of Sciences (DSc) degree by the University of Southampton (2004) and Honorary Doctorates by the TU of Budapest (2009) and by the University of Edinburgh (2015). He is a Foreign Member of the Hungarian Academy of Sciences and a former Editor-in-Chief of the IEEE Press. He has served several terms as Governor of both IEEE ComSoc and of VTS. He has published 1900+ contributions at IEEE Xplore, 19 Wiley-IEEE Press books and has helped the fast-track career of $123 \mathrm{PhD}$ students. Over 40 of them are Professors at various stages of their careers in academia and many of them are leading scientists in the wireless industry. 